\title{
Evolution of specialization under non-equilibrium population dynamics
}

Tuomas Nurmi

Kalle Parvinen (parvinen@iiasa.ac.at)

\section{Approved by}

Ulf Dieckmann

Director, Evolution and Ecology Program

June 2015

Interim Reports on work of the International Institute for Applied Systems Analysis receive only limited review. Views or opinions expressed herein do not necessarily represent those of the Institute, its National Member Organizations, or other organizations supporting the work. 


\title{
Evolution of specialization under non-equilibrium population dynamics
}

\author{
Tuomas Nurmi ${ }^{*, a}$, Kalle Parvinen ${ }^{\mathrm{a}, \mathrm{b}}$ \\ ${ }^{a}$ Department of Mathematics, FIN-20014 University of Turku, Finland \\ ${ }^{b}$ Evolution and Ecology Program, International Institute for Applied Systems Analysis, \\ A-2361 Laxenburg, Austria
}

\begin{abstract}
We analyze the evolution of specialization in resource utilization in a mechanistically underpinned discrete-time model using the adaptive dynamics approach. We assume two nutritionally equivalent resources that in the absence of consumers grow sigmoidally towards a resource-specific carrying capacity. The consumers use resources according to the law of mass-action with rates involving trade-off. The resulting discrete-time model for the consumer population has over-compensatory dynamics. We illuminate the way non-equilibrium population dynamics affect the evolutionary dynamics of the resource consumption rates, and show that evolution to the trimorphic coexistence of a generalist and two specialists is possible due to asynchronous non-equilibrium population dynamics of the specialists. In addition, various forms of cyclic evolutionary dynamics are possible. Furthermore, evolutionary suicide may occur even without Allee effects and demographic stochasticity.
\end{abstract}

Key words: Adaptive dynamics, Resource utilization, Trade-off, Specialist, Generalist, Evolution, Local adaptation

\section{Introduction}

Evolution of life history traits interacts with population dynamics. Especially well this interplay is known in the case of evolution of dispersal, where non-equilibrium population dynamics may forge dispersal and even enable evolutionary branching of dispersal strategies, but, on the other hand, dispersal may stabilize population dynamics (Gyllenberg et al., 1993; Holt and McPeek, 1996; Parvinen, 1999; Ronce, 2007). However, recent results indicate that the type of population-dynamical attractor may affect the evolution of other life history traits as well (White et al., 2006; Hoyle et al., 2011). In this paper, we analyze the interplay between population dynamics and the evolution of

\footnotetext{
*Corresponding author.

Email address: tuomas.nurmi@utu.fi (Tuomas Nurmi)
} 
resource utilization using the adaptive dynamics approach (Metz et al., 1992; Geritz et al., 1998).

In order to enable reasonable evolutionary analysis, we base our model on individual level processes (Rueffler et al., 2006a). We first assume continuous-time resource-consumer dynamics within breeding seasons for consumers utilizing two alternative resources. With specialization between two substitutable resources, a trade-off is necessarily present: a consumer may utilize both resources, but the more efficiently it uses one resource, the less efficiently it is able to use the other.

Following Geritz and Kisdi (2004), we assume time-scale separation between the dynamics of the resources and those of the consumers. This enables us to obtain a discrete-time model for the consumer population between breeding seasons. Different within-season resource dynamics result in different discrete-time between-season dynamics for the consumer population. We commit the majority of our analysis using a model that, in the case of only one resource, equates to the discrete logistic model, which is known to exhibit a wide range of different population-dynamical attractors from equilibrium to chaos (e.g. Holmgren (1994)). For comparison, we present also results obtained from models that correspond to the Ricker (1954) model and Hassell (1975) model. In all of these models, the type of the population-dynamical attractor affects the evolutionary dynamics of the consumers' resource utilization strategies. On the other hand, different consumer strategies result in different types of population-dynamical attractors.

The family of models we study has been extensively studied in the case of equilibrium dynamics by Nurmi and Parvinen (2008) who found three qualitatively different evolutionary scenarios: evolution to a monomorphic specialist population, evolutionary branching resulting in the coexistence of two specialist strategies, and evolution to a monomorphic generalist population. This is in line with the majority of previous results (Levins, 1962, 1963; Meszéna et al., 1997; Ma and Levin, 2006; Rueffler et al., 2006b; Ravigné et al., 2009). Previous work on other traits has shown that, under non-equilibrium population dynamics, evolutionary branching may be possible also in such ecological scenarios that do not allow branching under equilibrium dynamics (Parvinen, 1999; White et al., 2006; Hoyle et al., 2011). Thus, non-equilibrium dynamics may result in enhanced biodiversity. In our model, evolutionary branching is possible already under equilibrium dynamics. However, non-equilibrium dynamics may still add in diversity by allowing a secondary evolutionary branching to occur, which results in the trimorphic coexistence of generalists and specialists. Furthermore, non-equilibrium dynamics may, in our model, result in evolutionary suicide (Ferrière, 2000; Parvinen, 2005).

In the presence of only one resource, it is possible to investigate the evolution of the rate at which the consumer uses the resource. In this case, there are no costs involved in the utilization of the sole resource. Thus, in most cases, there is selection for ever increasing values of this rate until a physical maximum is reached. It is also possible that the resource population collapses or even goes extinct, e.g., because of Allee-effects or demographic stochasticity. This in turn 
can cause the extinction of the consumer population, i.e. evolutionary suicide.

In our model with logistic dynamics, too intensive use of resources can cause their collapse and thus the evolutionary suicide of the consumer population even in the absence of of Allee-effects and demographic stochasticity.

There are several models where the ecological coexistence of a generalist and two specialists is possible (Wilson and Yoshimura, 1994; Kisdi, 2002; Abrams, 2006b). However, such coexistence may be evolutionarily unstable. Even more rarely is such trimorphic coexistence evolutionarily attainable, i.e. reachable from an initially monomorphic population when mutations are assumed small. Egas et al. (2004) showed that evolution always destroys the trimorphic coexistence in the model of Wilson and Yoshimura (1994). Furthermore, Egas et al. (2004) showed that even in moderately modified versions of this model, evolution to the trimorphic coexistence is possible only in an extremely narrow parameter domain. Abrams (2006a) showed that, if the resource dynamics fluctuate asynchronously and the time consumers need to handle the resources is taken into account, evolution to the trimorphic coexistence is possible and, in the parameter domain where the ecological coexistence is possible, even plausible. Abrams (2006a) deduced that asynchrony in the resource dynamics gives generalists an advantage due to reduced variance in resource intake. This advantage may, however, disappear when the generalists become more common since this may have a synchronizing effect on the resource dynamics. In spatially heterogeneous models with global dispersal, evolution to the trimorphic coexistence is not possible under equilibrium dynamics (Nurmi and Parvinen, 2008; Nurmi et al., 2008) when only specialization can evolve, but recently Nurmi and Parvinen (2011) showed that the joint evolution of specialization and dispersal may result in the coexistence of an abundantly dispersing generalist and two scarcely dispersing specialists (see also Kisdi (2002)). Furthermore, when the resources are spatially aggregated, also distance-limited dispersal may enable evolution to the trimorphic coexistence where generalists live in the habitat boundaries (Debarre and Lenormand, 2011; Karonen, 2011).

In this paper, we integrate the ideas concerning the adaptive dynamics under non-equilibrium population dynamics (Parvinen, 1999; White et al., 2006; Hoyle et al., 2011) and the idea that asynchronous resource dynamics may enable coexistence of the specialists and generalists (Abrams, 2006a,b) with the mechanistic modeling approach used by Nurmi and Parvinen $(2008,2011)$ to analyze the evolution of specialization.

\section{Model and methods}

Following Geritz and Kisdi (2004), we assume that consumers hatch at the beginning of a breeding season and use resources to produce eggs that also encounter mortality during the breeding season. At the end of the season, all adults perish and only a fraction of the eggs survives to the following season.

In the absence of consumers, continuous-time within-season dynamics of resource $i$ are determined by the logistic differential equation with carrying capacity $K_{i}$, i.e., 


$$
\dot{R}_{i}=\alpha_{i}\left(1-\frac{R_{i}}{K_{i}}\right) R_{i},
$$

where $R_{i}$ denotes the density of the resource and $\alpha_{i}>0$ denotes the resource renewal rate. Different resources affect each other only via shared consumers. We assume that between breeding seasons resource populations recover to their carrying capacities independent of the usage during previous seasons.

The consumers use resources according to the law of mass action. The consumer individuals are all identical except for the specialization strategy $s \in[0,1]$ that affects only the resource consumption rates. An individual with strategy $s$ uses resource 1 with rate $\beta(s)$ and resource 2 with rate $\beta(1-s)$. The resource consumption (or trade-off) function $\beta$ is an increasing function with $\beta(0)=0$ and $\beta(1)=1$. Thus, case $s=0$ corresponds to a devoted specialist using only resource 2 and case $s=1$ to a devoted specialist using only resource 1 . Case $s=0.5$ corresponds to an unbiased generalist. For example, if a consumer population is monomorphic with strategy $s$ and population size $x$, the dynamics of resource 1 are $\dot{R}_{1}=\alpha_{1}\left(1-\frac{R_{1}}{K_{1}}\right) R_{1}-\beta(s) R_{1} x$. Within season, consumers produce eggs with rate proportional to their resource usage. The consumer population in the following season consists only of eggs that survive the winter and hatch.

When we, furthermore, assume that the resource dynamics are fast compared to the consumer dynamics such that the resources are always at the quasiequilibrium determined by the consumer population sizes and strategies, we finally obtain the following logistic-type difference equation for the consumer between-season dynamics (Geritz and Kisdi (2004), see also Nurmi and Parvinen (2008)):

$$
\begin{aligned}
x_{n+1} & =\lambda_{1} K_{1} \beta(s) x_{n} \max \left(0,1-\frac{\beta(s)}{\alpha_{1}} x_{n}\right) \\
& +\lambda_{2} K_{2} \beta(1-s) x_{n} \max \left(0,1-\frac{\beta(1-s)}{\alpha_{2}} x_{n}\right),
\end{aligned}
$$

where $\lambda_{i} \in \mathbb{R}_{+}$are compound parameters that depend on the details of the within season dynamics (Geritz and Kisdi, 2004).

In order to illuminate the differences between specialists and generalists we assume that the resources are nutritionally equivalent $\left(\lambda_{1}=\lambda_{2}=\lambda\right)$ and renew in equal rate $\left(\alpha_{1}=\alpha_{2}=\alpha\right)$. In this case, both $\lambda$ and $\alpha$ can be scaled out and, for several consumers, equation (2) takes form

$$
\begin{aligned}
x_{n+1}^{(j)} & =K_{1} \beta\left(s^{(j)}\right) x_{n}^{(j)} \max \left(0,1-\sum_{i=1}^{k} \beta\left(s^{(i)}\right) x_{n}^{(i)}\right) \\
& +K_{2} \beta\left(1-s^{(j)}\right) x_{n}^{(j)} \max \left(0,1-\sum_{i=1}^{k} \beta\left(1-s^{(i)}\right) x_{n}^{(i)}\right) \\
& =f\left(s^{(j)}, S, X_{n}\right) x_{n}^{(j)}
\end{aligned}
$$

where $f\left(s, S, X_{n}\right)$ is the fecundity of a strategy $s$ individual when strategies $S=$ $\left(s^{(1)}, s^{(2)}, \ldots, s^{(k)}\right)$ are present with population sizes $X_{n}=\left(x_{n}^{(1)}, x_{n}^{(2)}, \ldots, x_{n}^{(k)}\right)$. A rare mutant with strategy $s^{\text {mut }}$ and negligible population size $X_{n}^{\text {mut }}$ will grow 
according to $x_{n+1}^{\text {mut }}=f\left(s^{\text {mut }}, S, X_{n}\right) x_{n}^{\text {mut }}$. Under equilibrium dynamics, we can determine the fitness of a rare mutant in the environment set by the residents, in the spirit of Metz et al. (1992), as

$$
r\left(s^{\text {mut }}, S, X^{*}\right)=\ln \left(f\left(s^{\text {mut }}, S, X^{*}\right)\right),
$$

where $X^{*}$ denotes the vector of the equilibrium population sizes of the resident strategies. Under non-equilibrium dynamics, the calculation of the fitness function is more complicated: Assume that the resident population comprising strategies $S=\left(s^{(1)}, s^{(2)}, \ldots, s^{(k)}\right)$ has settled to an attractor $X=$ $\left(X_{1}, X_{2}, \ldots X_{n}, \ldots\right)$, where each $X_{n}$ is the vector of the population sizes at time $n$ as above. Then

$$
r(s, S, X)=\lim _{t \rightarrow \infty} \ln \left(\sqrt[t]{\prod_{i=1}^{t} f\left(s, S, X_{i}\right)}\right)=\lim _{t \rightarrow \infty} \frac{1}{t} \sum_{i=1}^{t} \ln \left(f\left(s, S, X_{i}\right)\right) .
$$

If $r(s, S, X)>0$, a mutant with strategy $s$ is able to invade the resident population. In practice, it is possible to calculate fitness only in the case of $p$-periodic resident population dynamics. In this case,

$$
r(s, S, X)=\frac{1}{p} \sum_{i=1}^{p} \ln \left(f\left(s, S, X_{i}\right)\right) .
$$

Some analytic results can be derived even without specifying the resource consumption function $\beta$ (Nurmi and Parvinen, 2008). However, our results mostly rely on the numerical analysis of equations (3) and (4) together with evolutionary simulations. In the numerical explorations, we use

$$
\beta(s)=\frac{1-e^{-\theta s}}{1-e^{-\theta}}, \quad \theta \neq 0 .
$$

This formula is not defined for $\theta=0$, but since $\lim _{\theta \rightarrow 0} \beta(s)=s$ it is natural to define $\beta(s)=s$ when $\theta=0$. The trade-off parameter $\theta$ determines whether the resource consumption function $\beta$ is convex $(\theta<0)$, concave $(\theta>0)$, or linear $(\theta=0)$. In the case of concave resource consumption function, the resource consumption function increases deceleratingly. This case is sometimes referred as the case of weak trade-off since a generalist can use resources more efficiently than a linear combination of the two specialists $\left(\beta(0.5)>\frac{\beta(0)+\beta(1)}{2}\right)$. Analogously, in the case of convex resource consumption function, the resource consumption function increases acceleratingly (strong trade-off, $\beta(0.5)<\frac{\beta(0)+\beta(1)}{2}$ ). In the terminology used by, e.g., White et al. (2006) and Hoyle et al. (2011), the case of concave resource consumption function corresponds to a trade-off with accelerating costs, and the case of convex resource consumption function corresponds to a trade-off with decelerating costs.

The resource consumption function is the only ingredient in our model that has no mechanistic interpretation. We use negative values of $\theta$ to phenomenologically model the situations where there is an additional cost of generalism, and 
positive values of $\theta$ to model those situations where there is an additional benefit of generalism. The linear resource consumption function $(\beta(s)=s, \theta=0)$ is an important special case since it can be interpreted, for example, as the search time allocation between the two resources. With the formulation (5), we obtain resource consumption functions that are almost similar to the case with $\beta(s)=s^{\theta}$, but avoid artificial singularities in the borders of the strategy space.

The derivative of $r(s, S, X)$ with respect to the mutant strategy $s$ (fitness gradient), determines the direction of evolution in a monomorphic population. The points where the fitness gradient vanishes are called evolutionarily singular strategies. There exists no directional evolution at a singular strategy. If evolution, in a neighborhood of a singular strategy, directs towards (or away from) this strategy, it is called evolutionarily attracting (or repelling) strategy. If no other nearby strategy cannot invade the resident population with this strategy, it is called an evolutionarily stable strategy (ESS, Maynard Smith and Price (1973)). In the case of frequency-dependent selection, however, it is possible that an evolutionarily attracting singular strategy can be invaded by any other nearby strategy. In this case, evolutionary branching occurs, i.e., the population splits into two distinct morphs that start to evolve further apart from each other. Evolutionary attractiveness and stability are independent properties, and all combinations are possible. For more information on singular strategies and their classification see Geritz et al. (1998).

Above, we assumed that the resources are equivalent, i.e. $\lambda_{1}=\lambda_{2}$ and $\alpha_{1}=\alpha_{2}$. If we, furthermore, assume that $K_{1}=K_{2}$, the environment becomes completely symmetric with respect to the resources. In a symmetric environment, the unbiased generalist strategy $s=0.5$ is always singular. We analyze mainly the case of symmetric environments since in this case it is easy to illuminate the differences between specialists and generalists and to observe how changes in the other ecological parameters affect the evolutionary dynamics.

\section{Evolution of specialization in the logistic model}

\subsection{Evolution of specialization under equilibrium population dynamics}

In our model, the trade-off parameter $\theta$ dominates the evolutionary dynamics. Under equilibrium population dynamics, there are only three qualitatively different evolutionary scenarios: if $\theta$ is assumed to have high enough values, the evolution of a monomorphic population directs towards generalism, and if low enough values, towards specialism. In other words, concave resource consumption function promotes generalism whereas strongly convex resource consumption function promotes specialism (compare with Nurmi and Parvinen (2008, 2011)). In the intermediate case with weakly convex resource consumption function, a monomorphic population evolves towards generalism where evolutionary branching takes place. Figure 1 illustrates evolutionary simulations from all different evolutionary scenarios possible under equilibrium population dynamics.

When the parameter values are such that the population shows equilibrium dynamics for all specialization strategies, we find the evolutionary bifurcation 


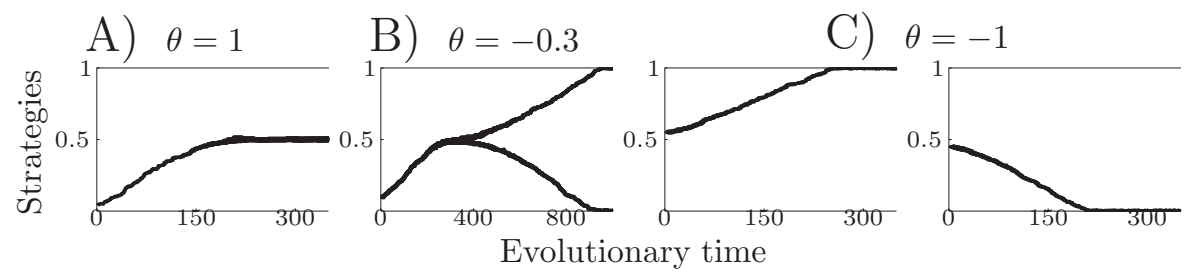

Figure 1: Evolutionary scenarios under equilibrium population dynamics.

Strategies present in the population as a function of the evolutionary time. One unit of evolutionary time corresponds to one loop of the simulation procedure depicted in the Appendix. Thus, it is only applicable for comparison between different simulations using the same procedure.

Panel A: Concave resource consumption function - Evolution leads to generalism.

Panel B: Weakly convex resource consumption function - Evolution of a monomorphic population leads to generalism where evolutionary branching takes place. The evolution of a dimorphic population leads to the combination of the two devoted specialists.

Panel C: Strongly convex resource consumption function - Evolution leads to the nearest devoted specialist strategy.

Other parameter values: $K_{1}=K_{2}=1.5, \alpha_{1}=\alpha_{2}=1, \lambda_{1}=\lambda_{2}=1$.

diagrams illustrated in Figure 2. The generalist strategy turns from a branching point to an evolutionarily stable strategy at $\theta=0$ where the resource consumption function turns from convex to concave. A simple calculation shows that under equilibrium population dynamics this is a rather general result (Nurmi and Parvinen, 2008). Under non-equilibrium dynamics, this relation no longer holds (See e.g. Figure 9A). Corresponding results have been obtained also by White et al. (2006); Hoyle et al. (2011). The parameter domains colored black in Figure 2 are such that the population is not viable due to low resource intake: when the resources are scarce, the additional cost of generalism (negative $\theta$ ) may cause extinction. When some strategies in the strategy space are not viable, it is worthwhile to consider, whether evolutionary suicide is possible. Evolutionary suicide may occur when evolution drives the strategy of the evolving population towards the unviable part of the strategy space. This is possible when mutations that are beneficial at the individual level are harmful at the population level (e.g. "tragedy of commons" (Hardin, 1968)). At the extinction boundary, it is possible that the resident population is invaded by a "kamikaze mutant" that can outcompete the other strategies but is not viable alone and thus the species dies out. However, if the population-dynamical attractor, as a function of the resident strategy, approaches zero continuously, the resident population is almost absent in the neighborhood of the extinction boundary. This means that the invasion fitness of a mutant is the same as the fitness in a virgin environment. Thus, only mutants that are viable alone are able to invade, and evolutionary suicide is impossible. Therefore, a necessary condition for evolutionary suicide is that the population-dynamical attractor drops discontinuously from a viable non-trivial attractor to the trivial attractor corresponding to extinction. This result has been proven algebraically for a wide class of models by Gyllenberg et al. (2002), see also Parvinen (2005). 

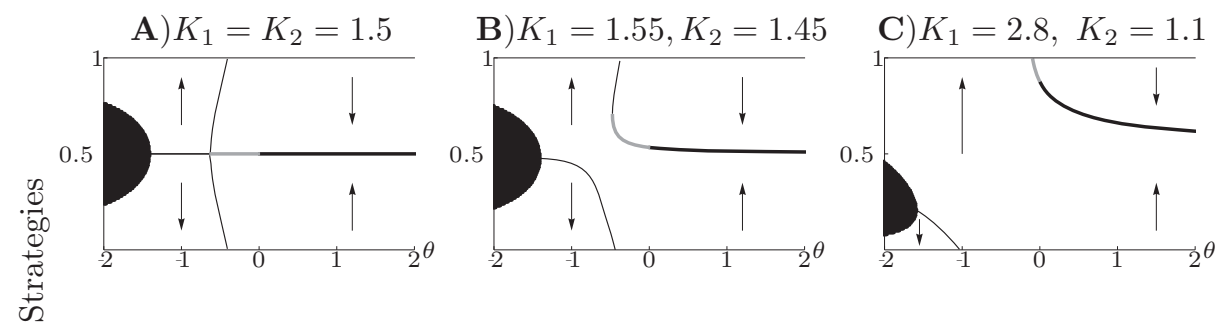

Evolutionary time

Figure 2: Evolutionary bifurcation diagrams in the case of equilibrium population dynamics. Singular strategies as a function of the trade-off parameter $\theta$. Thin black curve indicates evolutionary repellors, thick grey curve branching points and thick black curve evolutionarily stable strategies. The arrows indicate the direction of evolution in a monomorphic population. In the black-colored parameter domain, the population is not viable due to low resource intake. Other parameter values: $\alpha_{1}=\alpha_{2}=1, \lambda_{1}=\lambda_{2}=1$.

In the case of equilibrium population dynamics (Figure 2), the transition to extinction takes place continuously: The equilibrium population size decreases continuously to zero when the specialization strategy approaches the extinction boundary (see Figure 4A). The continuous transition to extinction guarantees that the extinction boundary is evolutionarily repelling, and thus evolutionary suicide is not possible in the neighborhood of this parameter domain.

\subsection{Non-equilibrium population dynamics}

If resource carrying capacities have large values, the population dynamics may be periodic or even chaotic. Furthermore, the population-dynamical attractors may be qualitatively different for consumers using different strategies (see Figure 4). For a strategy $s=1$ consumer utilizing solely resource 1 , the type of the population-dynamical attractor is determined solely by the parameter $K_{1}$ similarly to the logistic recurrence equation $x_{n+1}=K_{1} x_{n}\left(1-x_{n}\right)$. If $0<K_{1}<1$, the corresponding specialist with strategy $s=1$ is not viable. If $1<K_{1}<3$, a monomorphic specialist population shows equilibrium dynamics. If $3<K_{1}<4$, a monomorphic specialist population shows periodic or chaotic dynamics. If $4<K_{1}$, a devoted specialist with $s=1$ is not viable, because the population growth is fast enough to exhaust resource 1 (see equation (2)). Furthermore, note that the trade-off parameter $\theta$ does not affect the population dynamics of a monomorphic population of devoted specialist since $\beta(1)=1$ independent of $\theta$. Analogous results hold for strategy $s=0$ specialists.

For an unbiased generalist strategy (which is singular if $K_{1}=K_{2}$ ), the attractor type is analogously determined by the sum $\beta(0.5)\left(K_{1}+K_{2}\right)$. Figure 3 illustrates the population-dynamical attractors of the unbiased generalist $(s=$ $0.5)$ population as a function of the trade-off parameter $\theta$. In panel A, the generalist population is unviable for low values of $\theta$ due to low resource intake (low resource carrying capacities and the additional cost of generalism) whereas in panel $\mathrm{B}$, the population is unviable for large values of $\theta$ since both resources 


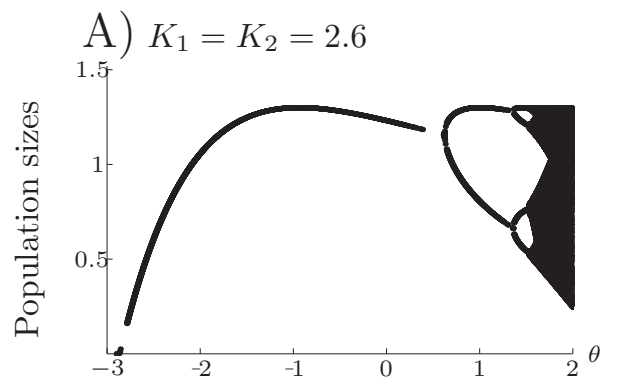

B) $K_{1}=K_{2}=3.8$

Figure 3: Population-dynamical attractors of an unbiased generalist population $(s=0.5)$ as a function of the trade-off parameter $\theta$. The panels correspond to those of Figure 5. Other parameter values: $\alpha_{1}=\alpha_{2}=1, \lambda_{1}=\lambda_{2}=1$.

become exhausted because high resource carrying capacities and the additional benefit of generalism result in overly fast consumer population growth.

Figure 4 illustrates the population-dynamical attractors as a function of the specialization strategy $s$. In Figure $4 \mathrm{~A}$, the transitions to extinction at $\theta \approx 0.36$ and $\theta \approx 0.74$ occur continuously and thus evolutionary suicide is not possible, whereas in Figure $4 \mathrm{D}$, these transitions at $\theta \approx 0.43$ and $\theta \approx 0.57$ occur discontinuously, which means that evolutionary suicide may be possible in this setting. Below we show that evolutionary suicide actually happens. Figures 4B and $4 \mathrm{C}$ illustrate that the resource consumption strategy may affect population dynamics in a variety of ways.

\subsection{Evolution of specialization under non-equilibrium population dynamics}

Figure 5 illustrates the evolutionary dynamics in the case of possibly periodic or chaotic population dynamics. It shows the evolutionary singular strategies as a function of the trade-off parameter $\theta$ together with the endpoints of the corresponding evolutionary simulations (see the Appendix for the description of the simulation procedure). In Figure 5A, devoted specialists have equilibrium population dynamics, whereas in Figure $5 \mathrm{~B}$, they have chaotic dynamics.

We are not aware of any algebraic means for calculating fitness under chaotic population dynamics. The population-dynamical route to chaotic dynamics takes place via a series of period-doubling bifurcations (see Figures 3 and 4).

For the population-dynamical attractors of period $1,2,4,8$ or 16 we base our analysis on fitness gradient using equation (4). In (the neighborhood of) the parameter domain where population dynamics are chaotic, this method is not applicable. There we are forced to rely solely on evolutionary simulations. Such parameter domains are colored grey in Figure 5. Note, that in a dimorphic or polymorphic population, the parameter domains with chaotic population dynamics may be completely different. Note also that the search for attractors could be extended, but the increase in the size of the analytically treatable parameter domain would be rather small and the increase in computational time would be substantial. 

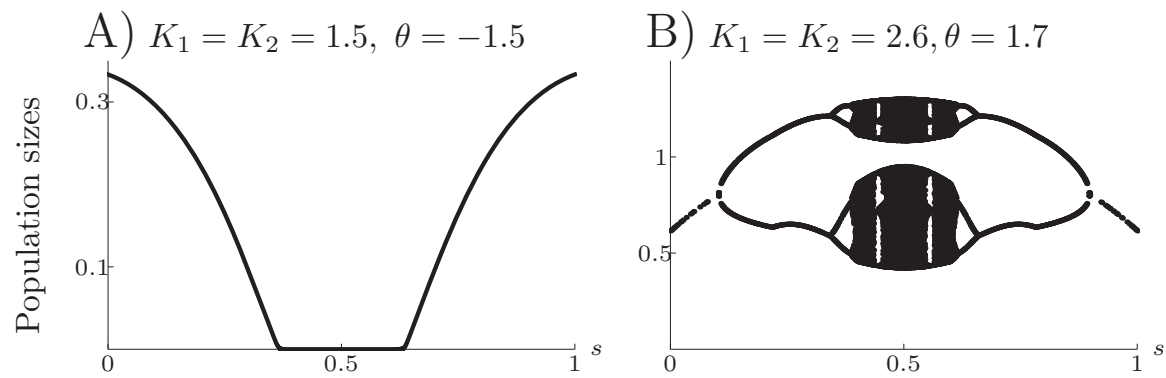

C) $K_{1}=K_{2}=3.8, \theta=-1$

D) $K_{1}=K_{2}=3.8, \theta=1$
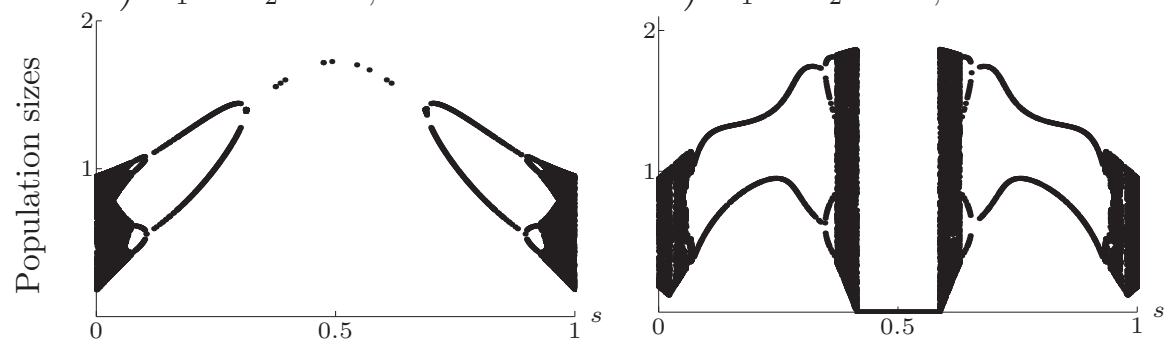

Figure 4: Population-dynamical attractors as a function of the specialization strategy $s$.

Panel A: Continuous transition to extinction when the resource carrying capacities are low such that, due to the additional cost of generalism, the resource intake by generalists is not high enough to maintain viability of the population (cf. Figure 2).

Panel B: Specialists have equilibrium population dynamics but, due to the additional benefit of generalism $(\theta>0)$, generalists have chaotic population dynamics (cf. Figure 5A).

Panel C: Specialists have chaotic population dynamics but the usage of two resources together with the additional cost of generalism stabilizes the population dynamics (cf. Figure 5B).

Panel D: Discontinuous transition to extinction. High resource carrying capacities and additional benefit of generalism accelerate the growth of the consumer population. Finally resources are exhausted and the consumer population goes abruptly extinct. Note, that biased usage of two resources may still stabilize population dynamics (cf. Figure 5B).

Other parameter values $\alpha_{1}=\alpha_{2}=1, \lambda_{1}=\lambda_{2}=1$. 
All the simulations illustrated in Figure 5 start with an initially monomorphic population with a random initial strategy and population size. When an evolutionary simulation ends in a monomorphic population, we illustrate this endpoint using a $\diamond$-sign. When evolutionary branching takes place, we illustrate the strategies present at the end of the simulation using $*$-signs. Since all the simulations are run over only a finite time, it is possible that some of them have not yet reached an evolutionarily stable strategy.

The parameter domains colored black in Figure 5 are such that the population is not viable. In Figure 5A this unviability is caused by low resource intake (see Figure 4A), and as explained above, evolutionary suicide is not possible. In Figure 5B unviability occurs since high resource carrying capacities together with the additional benefit of generalism result in population growth fast enough to exhaust the resources. As assumed in the model description, the resources recover next time unit after being exhausted. The consumer population, however, cannot recover, and extinction results. In this case, the transition from viable parameter domain to extinction occurs discontinuously (see Figure $4 \mathrm{D})$ and evolutionary suicide occurs whenever $\theta \gtrsim 0.3$. In Figures $5 \mathrm{~B}$ and $9 \mathrm{~B}$ the $\dagger$-sign at the boundary of this black area illustrates the last viable strategy before extinction in an evolutionary simulation.

When the resource consumption function is sufficiently convex (the trade-off parameter $\theta$ low), the evolution of specialization leads to a population comprising one or two devoted specialist strategies both under equilibrium population dynamics (Figures 2 and 5A) and under non-equilibrium population dynamics (Figure 5B). For weakly convex resource consumption function $(-1 \lesssim \theta \lesssim 0$ in Figure 5), however, the evolutionary dynamics differ qualitatively between the cases with equilibrium (panel A) and non-equilibrium (panel B) population dynamics. Under equilibrium population dynamics, the evolution of a monomorphic population leads to generalism where evolutionary branching takes place and finally evolution ends in a combination of the two devoted specialist strategies. Under non-equilibrium population dynamics, as well, the evolution of a monomorphic population leads to generalism and evolutionary branching takes place. However, after branching the evolution of the dimorphic population does not lead to the combination of the devoted specialist strategies. Instead, either another evolutionary branching results in the trimorphic coexistence of a generalist and two devoted specialists, or the population remains dimorphic, but does not evolve to the coexistence of two devoted specialists. Next we discuss these two cases in detail.

\subsection{Dimorphic evolution of specialization under non-equilibrium population dy-} namics

When the strategies $s^{(1)}$ and $s^{(2)}$ in a dimorphic population are symmetric $\left(s^{(1)}=1-s^{(2)}\right)$, and the environment is symmetric $\left(K_{1}=K_{2}\right)$, then it follows directly from equation (3), that the diagonal $x^{(1)}=x^{(2)}$ in the populationdynamical state-space is invariant, i.e., if $x_{n}^{(1)}=x_{n}^{(2)}$, then also $x_{n+1}^{(1)}=x_{n+1}^{(2)}$. Such an in-phase orbit is called a symmetric orbit. It is possible to show algebraically (See the Appendix) that in such a case, the dimorphic population 


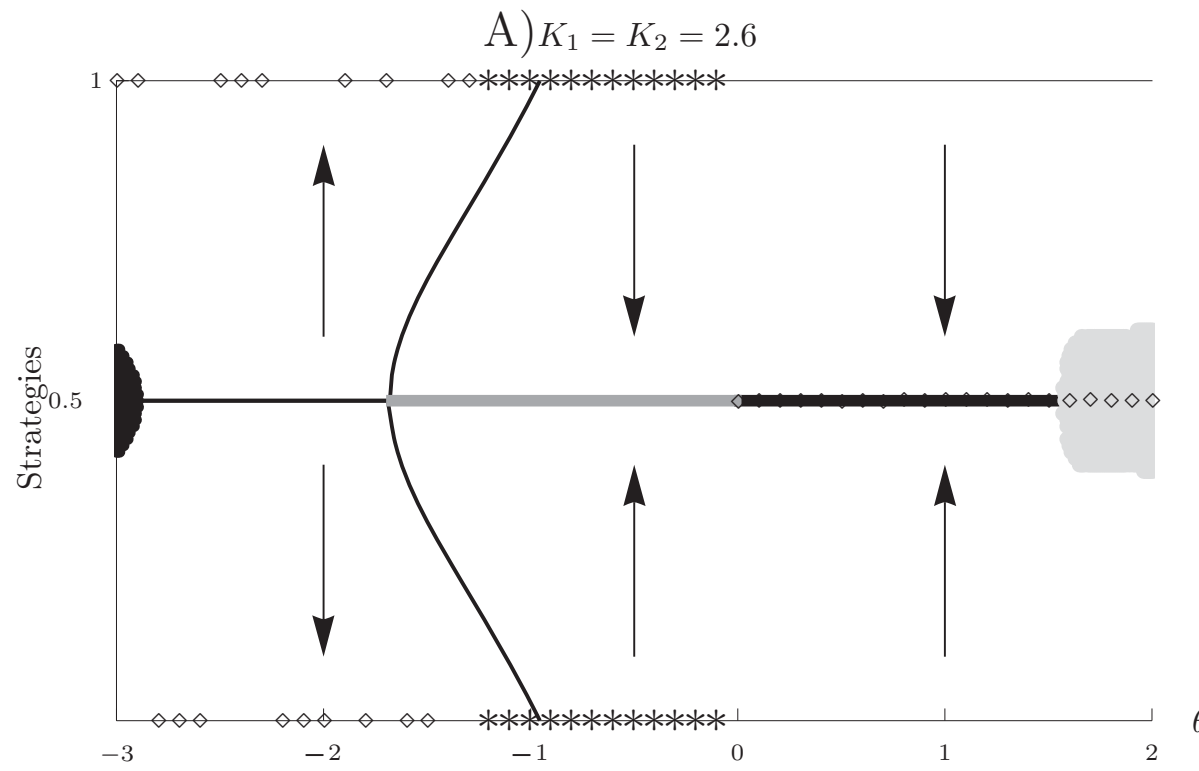

B) $K_{1}=K_{2}=3.8$

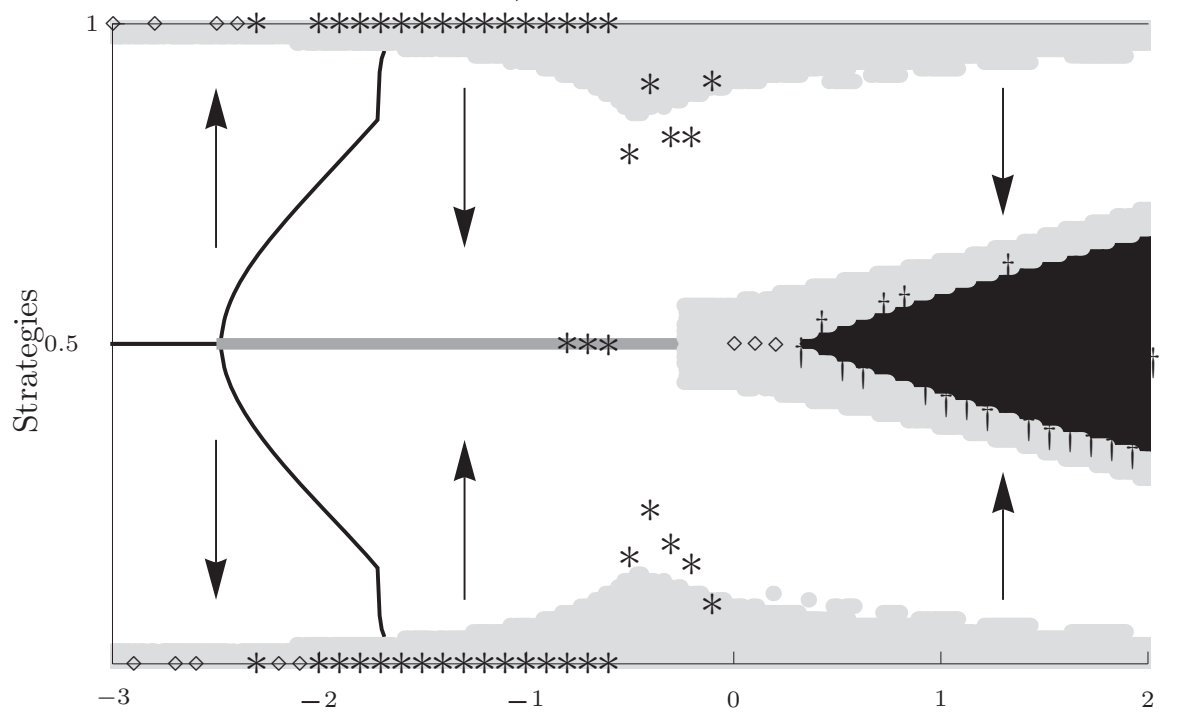

Figure 5: Evolutionary bifurcation diagrams in the case of possibly non-equilibrium population dynamics. Singular strategies and the endpoints of evolutionary simulations as a function of the trade-off parameter $\theta$. Thin black curve indicates evolutionary repellors, thick grey curve branching points and thick black curve evolutionarily stable strategies. The arrows indicate the direction of evolution in a monomorphic population. In the black-colored parameter domain, the population is not viable. In the grey-colored parameter domain, the monomorphic population dynamics are (nearly) chaotic. If an evolutionary simulation ends in a monomorphic population, the end-strategy is denoted by $\diamond$. If it ends in a dimorphic or polymorphic population, the strategies comprising the endpoint are denoted by $*$-signs. If evolutionary suicide occurs, the last viable strategy is denoted by $\dagger$-sign. The corresponding population dynamics are illustrated in Figures 23 and 4.

Panel A: Specialists have equilibrium population dynamics but high benefit of generalism enables non-equilibrium population dynamics for generalists when $\theta$ is sufficiently large.

Panel B: Specialists have chaotic population dynamics. Biased usage of two resources may stabilize population dynamics, but high benefit of generalism enables chaotic dynamics and even evolutionary suicide.

Other parameter values: $\alpha_{1}=\alpha_{2}=1, \lambda_{1}=\lambda_{2}=1$. 
1 always evolves towards the coexistence of the two devoted specialists given that the resource consumption function is convex. This result holds also for other models with similar underpinnings, e.g., the Ricker model (8).

When evolutionary branching occurs, the dimorphic population "inherits" its population-dynamical attractor from the preceding monomorphic population. For example, if the monomorphic population was on a two-periodic populationdynamical attractor, the dimorphic population is, immediately after branching, on an in-phase two-periodic orbit (See Geritz et al. (2002) for more information on attractor inheritance. Corresponding phenomenon has also been observed by Hoyle et al. (2011)). Thus, after evolutionary branching in a symmetric environment, the dimorphic population is always initially on a symmetric attractor. Therefore, the dimorphic population evolves towards the coexistence of the two devoted specialists, as long as the population-dynamical attractors remain symmetric. However, the symmetry of the population-dynamical attractors may be lost for several reasons:

- The symmetric population-dynamical orbit (equilibrium, cycle or chaotic) may become population-dynamically unstable. In this case, population switches to a new population-dynamical attractor with new, possibly different, evolutionary dynamics.

- Even though the symmetric population-dynamical orbit would remain population-dynamically stable, it can be chaotic. In such a case, stochastic mutations (although they are small) will eventually take the population dynamics away from the domain of attraction of the symmetric orbit, and again the population switches to another population-dynamical attractor.

- In the symmetric case $s^{(1)}=1-s^{(2)}$ the evolutionary forces acting on these strategies are also symmetric, which means that average evolutionary trajectories would be along $s^{(1)}=1-s^{(2)}$ to full specialism. Due to mutational stochasticity, the dimorphic population may evolve away from this trajectory. Furthermore, deviations from this trajectory may be enhanced by different evolutionary forces.

We have not found any means for algebraic analysis of the evolutionary dynamics in the case of asymmetric chaotic population-dynamical attractors. Therefore, we have to rely on evolutionary simulations. In symmetric environments, we have found three qualitatively different evolutionary scenarios for the dimorphic population:

- The population evolves to the coexistence of the two devoted specialists similarly to the case with equilibrium dynamics (Figure 1B).

- The population remains dimorphic, but does not evolve to devoted specialism. Results of such evolutionary simulations are illustrated in Figures 6 and 7 .

- Another evolutionary branching occurs and the population becomes trimorphic. One result of such an evolutionary simulation is illustrated in Figure 8 . 
In order to illuminate how the population dynamics affect the evolutionary dynamics, we need to illustrate the population-dynamical attractors during the evolutionary time together with the evolutionary tree in the strategy space. However, the evolutionary simulations we present here are never completely mutation limited. Instead, the population is, in practice, always polymorphic during the simulation. Therefore, in order to illustrate the population-dynamical attractor of the entire population, we need to calculate how much extant strategies use resources, which in turn allows us to calculate the availabilities of the resources. If strategies $\left(s^{(1)}, s^{(2)}, \ldots, s^{(k)}\right)$ are present at time unit $n$ with population sizes $\left(x_{n}^{(1)}, x_{n}^{(2)}, \ldots, x_{n}^{(k)}\right)$, then the availabilities $A_{1}(n)$ and $A_{2}(n)$ of the resources $R_{1}$ and $R_{2}$, respectively, are

$$
\begin{aligned}
& A_{1}(n)=K_{1} \max \left(0,1-\sum_{i=1}^{k} \beta\left(s^{(i)}\right) x_{n}^{(i)}\right) \\
& A_{2}(n)=K_{2} \max \left(0,1-\sum_{i=1}^{k} \beta\left(1-s^{(i)}\right) x_{n}^{(i)}\right) .
\end{aligned}
$$

When the population is on a non-equilibrium attractor, these availabilities fluctuate as the consumer population sizes fluctuate. Based on these availabilities, it is often possible to deduce the type of the population-dynamical attractor of the consumer population as a whole. For example, if the population is on a two-periodic in-phase orbit (symmetric attractor), the sum of the resource availabilities takes two different values on the population-dynamical attractor whereas their difference is close to zero. If the population is on a two-periodic out-of-phase orbit (asymmetric attractor), the differences alternate between a positive and a negative value on the population-dynamical attractor whereas the sum remains virtually constant. More generally: the more asynchronous are the resource fluctuations the larger are the absolute values of the differences in the resource availabilities.

Figure 6 illustrates the result of an evolutionary simulation ending in a singular dimorphic strategy pair (not devoted specialists) under periodic population dynamics. Figure $6 \mathrm{~A}$ illustrates the strategies present during an evolutionary simulation: for each unit of the evolutionary time, we plot a black point to each strategy present at that time unit. Panels B-D illustrate the resource availabilities. At the end of each loop of the evolutionary simulation procedure (i.e. for each evolutionary time unit) we observe the strategies present $\left(s^{(1)}, s^{(2)}, \ldots, s^{(k)}\right)$ and their population sizes $\left(x_{0}^{(1)}, x_{0}^{(2)}, \ldots, x_{0}^{(k)}\right)$. The population-dynamical attractor $\left(\left(x_{n}^{(1)}, x_{n}^{(2)}, \ldots, x_{n}^{(k)}\right), n=0,1,2, \ldots\right)$, that corresponds to this unit of evolutionary time, can then be calculated iteratively using equation (3) (for 20 steps in Figure 6). Furthermore, we can calculate the corresponding resource availabilities $A_{1}(n)$ and $A_{2}(n)$ using equation (6) and calculate their sum $A_{1}(n)+A_{2}(n)$ and difference $A_{1}(n)-A_{2}(n)$ for each population-dynamical step. For each evolutionary time unit in Figure $6 \mathrm{~B}$, we plot a black point for each different sum of the resource availabilities observed on the population-dynamical attractor at the end of the corresponding loop of the evolutionary simulation. In Figure $6 \mathrm{C}$, we plot the difference of the resource availabilities in a similar way, and in Figure $6 \mathrm{D}$, we plot the availability $A_{1}$ of 
esource 1.

In Figure 6, evolutionary branching takes place while the population is on a two-periodic attractor. As a consequence, after branching the dimorphic population is on an in-phase two-periodic orbit, i.e. it is on a symmetric attractor. However, as the two branches specialize further, this population-dynamical attractor becomes unstable, and the population switches to a new, four-periodic out-of-phase orbit (which is asymmetric). This creates asynchrony to the availabilities of the resources, which in turn, benefits generalism and stops the evolution towards specialism. Finally, evolution leads to a dimorphic singular strategy pair $\left(s^{(1)} \approx 0.16488, s^{(2)} \approx 0.83512\right)$

Also Figure 7 illustrates the result of an evolutionary simulation, where evolution does not lead to the combination of the two devoted specialists. Contrary to the simulation illustrated in Figure 6, the population dynamics in Figure 7 are chaotic, which ensures that the symmetry of the attractors in the dimorphic population is lost almost immediately. However, the two branches continue to specialize further until $0.13 \lesssim s^{(1)} \lesssim 0.23$ in one branch and $0.77 \lesssim s^{(2)} \lesssim 0.87$ in the other. Once the strategies of the evolving population have reached this dimorphic intermediate strategy region, they remain there. However, the population does not settle to any evolutionarily singular strategy combination. When the population dynamics are chaotic, several (even infinitely many) different population-dynamical attractors may exist simultaneously, and furthermore, even small random mutations may induce population-dynamical attractor switchings. When the population-dynamical attractor changes, it is possible that also the evolutionary forces acting on the population change. This phenomena is exemplified also in Figure 6 where attractor switches from an in-phase orbit to an out-of-phase orbit halts the dimorphic evolution towards devoted specialism. Similar examples have also been observed for other traits (Parvinen, 1999; Dercole et al., 2002; White et al., 2006; Hoyle et al., 2011). In Figure 7, population-dynamical attractor switching sometimes affects the evolutionary dynamics such that the dimorphic population evolves to a new strategy combination within the same dimorphic intermediate strategy region. Chaotic population dynamics prevent us from analyzing these switchings in detail. Figures $7 \mathrm{~B}$ and $7 \mathrm{C}$ suggest that attractor switching occur rather frequently. However, from Figure 7A one can observe that only some of the seem to have evolutionary effects. This is natural, because an attractor switching may be evolutionarily ineffective, or it may be succeeded by another attractor switching that balances its effects.

In Figure 5B, we observe evolutionary dynamics described above (Figure $7)$ within the parameter interval $-0.585 \lesssim \theta \lesssim 0$. The evolutionary simulations lead to dimorphic populations where the strategies of the two branches do not evolve to the devoted specialism. They do not evolve to any singular strategy combination either. Instead, they remain in some intermediate strategy region (which naturally depends on the trade-off parameter $\theta$ ) and undergo infrequent evolutionary transitions within this strategy region caused by populationdynamical attractor switchings. Therefore in Figure 5B, the endpoints of evolutionary simulations do not form any clear pattern for $-0.585 \lesssim \theta \lesssim 0$. 


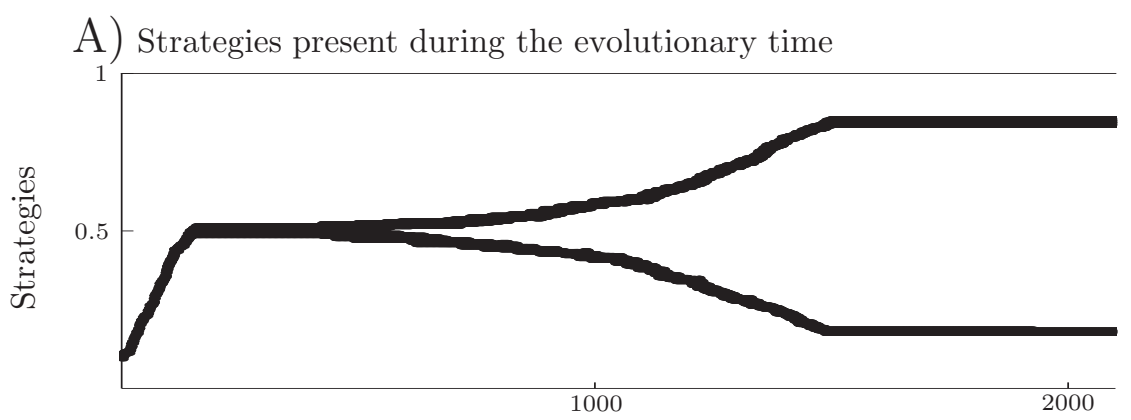

B) Sum of the resource availabilities

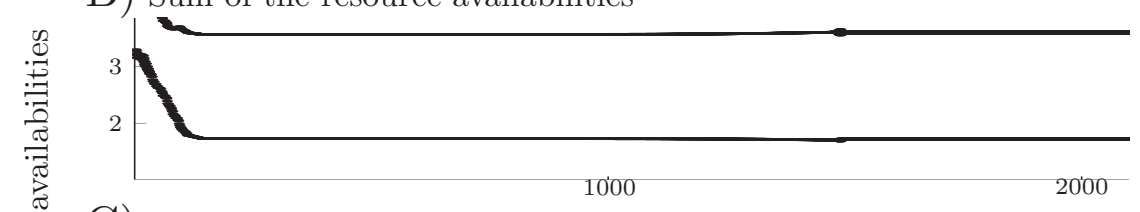

C) Difference between the resource availabilities

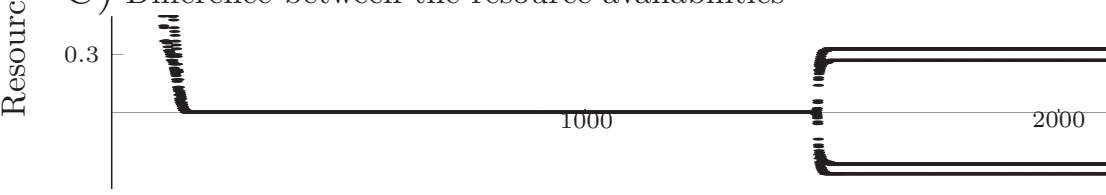

D) Availability $A_{1}$ of resource 1

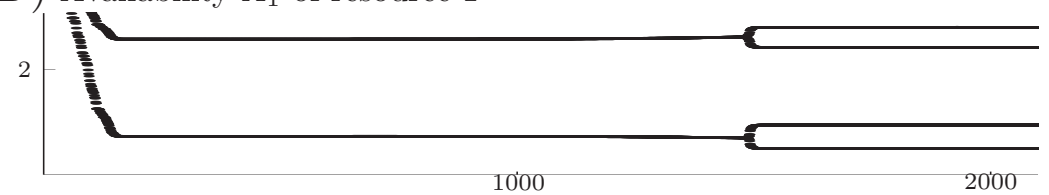

Evolutionary time

Figure 6: The result of an evolutionary simulation leading to a dimorphic singular strategy pair under periodic population dynamics.

Panel A: Strategies present in the population as a function of the evolutionary time. One unit of evolutionary time corresponds to one loop of the simulation procedure depicted in the Appendix. Thus, it is only applicable for comparison between different simulations using the same procedure.

Panels B, C, and D: Resource availabilities $A_{1}$ and $A_{2}$ as defined in equation (6) as a function of the evolutionary time. For each evolutionary time unit, Panel B illustrates the sum of the resources availabilities during each step on the population-dynamical attractor. Panel C illustrates the differences of the resource availabilities and panel D the availability of resource 1.

Parameter values: $K_{1}=K_{2}=3.5, \theta=-0.1, \alpha_{1}=\alpha_{2}=1, \lambda_{1}=\lambda_{2}=1$. 


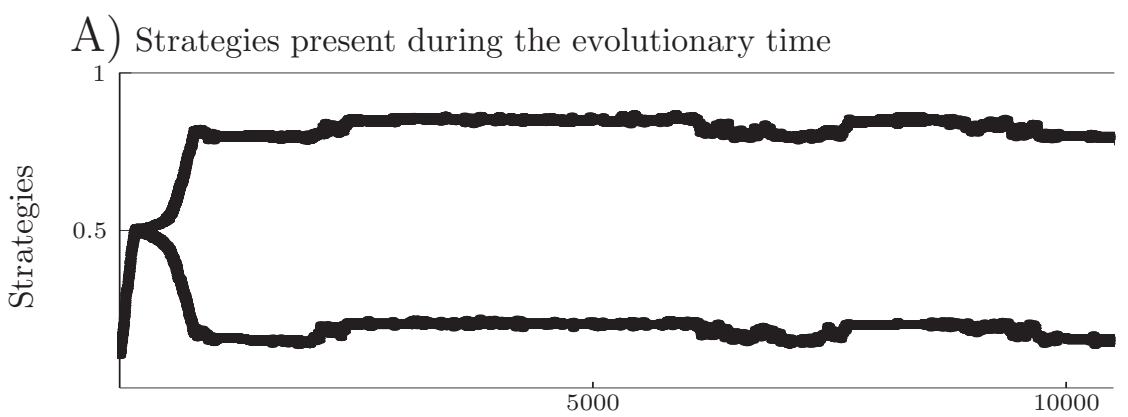

B) Sum of the resource availabilities

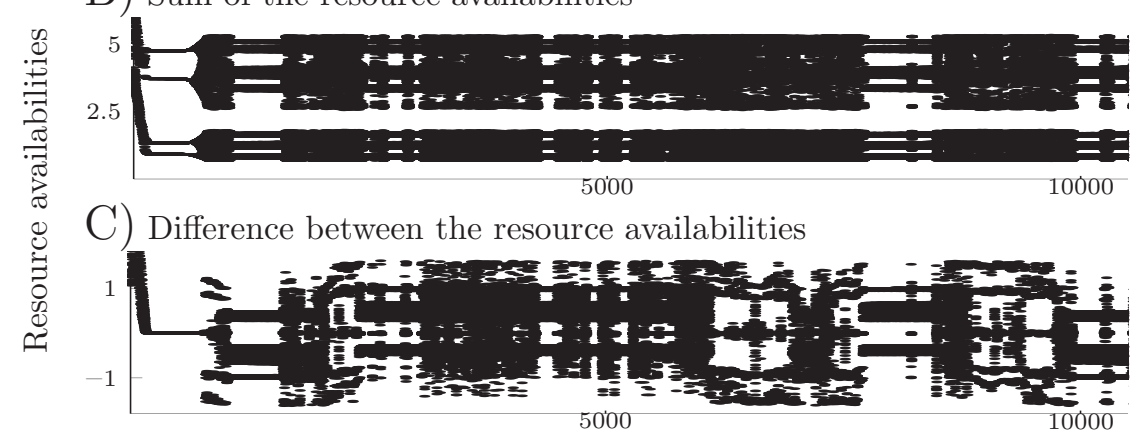

Evolutionary time

Figure 7: The result of an evolutionary simulation where a singular strategy combination is never reached because the population-dynamical attractor switchings induced by chaotic population dynamics affect evolutionary dynamics.

Panel A: Strategies present in the population as a function of the evolutionary time. One unit of evolutionary time corresponds to one loop of the simulation procedure depicted in the Appendix. Thus, it is only applicable for comparison between different simulations using the same procedure.

Panels B and C: Resource availabilities $A_{1}$ and $A_{2}$ as defined in equation (6) as a function of the evolutionary time. For each evolutionary time unit, Panel B illustrates the sum of the resources availabilities during each step on the population-dynamical attractor. Panel C illustrates the differences of the resource availabilities.

Parameter values: $K_{1}=K_{2}=3.8, \theta=-0.3, \alpha_{1}=\alpha_{2}=1, \lambda_{1}=\lambda_{2}=1$. 
In Figure $5 \mathrm{~B}$ with $-0.86 \lesssim \theta \lesssim-0.586$, evolution leads to the trimorphic coexistence of a generalist strategy and two devoted specialist strategies, as illustrated in Figure 8. In well-mixed populations, evolution to this coexistence has been shown possible when the resources fluctuate asynchronously (Abrams, 2006a). In our model, the resources have simple equilibrium dynamics in the absence of consumers. However, the availability of the resources is determined not only by the equilibrium value of the resource dynamics (resource carrying capacity) but also by the population sizes and strategies of the consumers utilizing the resources. When the population comprises two specialist branches that fluctuate asynchronously, a generalist strategy may be viable due to the reduced variance in the resource intake: due to the asynchronism in the fluctuations of the resource availabilities, at least one of the two resources will usually be available for the generalist whereas the specialists suffer frequently from low resource availability. Therefore, the higher the absolute value of the difference between the resource availabilities, the more likely the generalist strategy can coexists with the specialists.

Based on Figure 8, it is possible to deduce the population-dynamical route to the coexistence of a generalist and two specialists. The monomorphic population first evolves to generalism, where evolutionary branching occurs under periodic population dynamics. After branching, the population-dynamical attractors of the two branches are first symmetric (negligible difference between the resource availabilities) due to attractor inheritance (Geritz et al., 2002). As the branches specialize further, their population dynamics undergo a series of period-doubling bifurcations and finally their population dynamics looks rather chaotic. Meanwhile, the symmetry of the attractors is lost. However, the dimorphic population dynamics are not completely chaotic, and after a while, the population settles to an out-of-phase two-periodic orbit (the sum of the resource availabilities remains constant whereas the difference alternates between two values). On the out-of-phase orbit, the generalists can coexist with the specialists. The dimorphic population evolves to a singular strategy pair $\left(s^{(1)}=0, s^{(2)} \approx 0.919\right)$, which is an evolutionary branching point for strategy $s^{(2)} \approx 0.919$. Thus, second evolutionary branching starts slowly taking place. The out-of-phase orbit maintains its population-dynamical stability during the second branching and, finally, the population comprises the unbiased generalist strategy together with two devoted specialist strategies. Although we illustrate this phenomenon only for symmetric environments, it is present also in asymmetric environments. The generalist in this trimorphic coexistence, however, is the unbiased generalist only in symmetric environments.

\subsection{Evolution of specialization under non-equilibrium population dynamics and asymmetric environments}

Figure 9 illustrates the case where the environment is asymmetric and chaotic population dynamics are possible. In Figure $9 \mathrm{~A}$, chaotic population dynamics are exclusively due to the high carrying capacity of resource 1, while the carrying capacity of resource 2 is barely high enough to ensure the viability of the corresponding devoted specialist. In Figure 9B, the carrying capacity of resource 2 is 


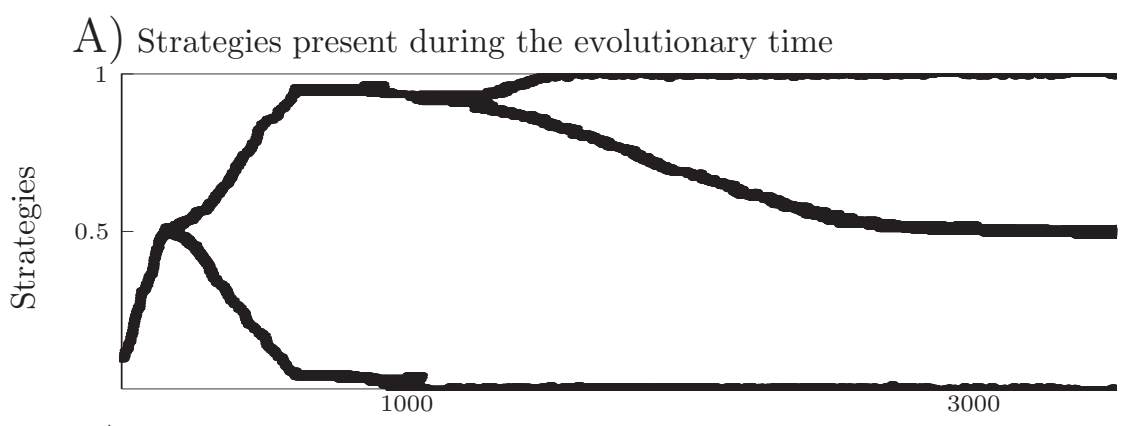

B) Sum of the resource availabilities

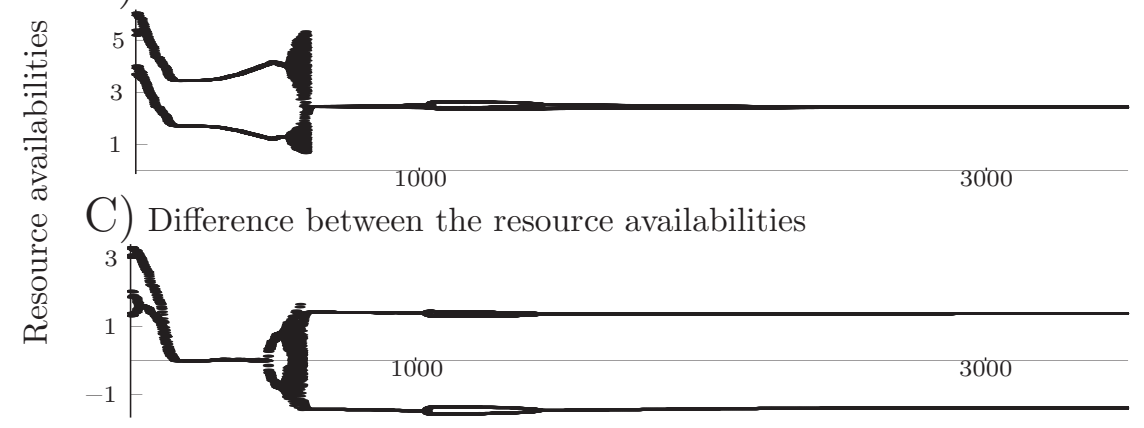

Evolutionary time

Figure 8: The result of an evolutionary simulation leading to the coexistence of generalist and specialists.

Panel A: Strategies present in the population as a function of the evolutionary time. One unit of evolutionary time corresponds to one loop of the simulation procedure depicted in the Appendix. Thus, it is only applicable for comparison between different simulations using the same procedure. Initial population is monomorphic practicing strategy $s=0.1$. Simulation ended in a trimorphic population practicing strategies $s_{1}=0, s_{2}=0.5$, and $s_{3}=1$.

Panels B and C: Resource availabilities $A_{1}$ and $A_{2}$ as defined in equation (6) as a function of the evolutionary time. For each evolutionary time unit, Panel B illustrates the sum of the resources availabilities during each step on the population-dynamical attractor. Panel C illustrates the differences of the resource availabilities.

Parameter values: $K_{1}=K_{2}=3.8, \theta=-0.72, \alpha_{1}=\alpha_{2}=1, \lambda_{1}=\lambda_{2}=1$. 

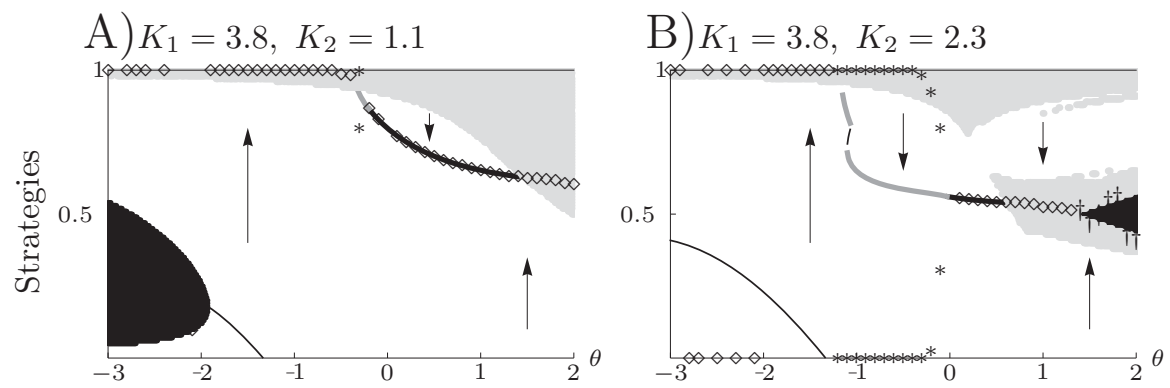

Figure 9: Evolutionary bifurcation diagrams in the case of possibly chaotic population dynamics and asymmetric environments. Singular strategies and endpoints of evolutionary simulations as a function of the trade-off parameter $\theta$. Thin black curve indicates evolutionary repellors, thick grey curve branching points and thick black curve evolutionarily stable strategies. The arrows indicate the direction of evolution in a monomorphic population. In the black-colored parameter domain, the population is not viable. In the grey-colored parameter domain, the monomorphic population dynamics are (nearly) chaotic. If an evolutionary simulation ends in a monomorphic population, the end-strategy is denoted by $\diamond$. If it ends in a dimorphic or polymorphic population, the strategies comprising the endpoint are denoted by $*$-signs. If evolutionary suicide occurs, the last viable strategy is denoted by $\dagger$-sign.

Other parameter values: $\alpha_{1}=\alpha_{2}=1, \lambda_{1}=\lambda_{2}=1$.

higher. This stabilizes the population dynamics when consumers are partially specialized on resource $1(0.65 \lesssim s \lesssim 0.85)$. On the other hand, in the cases with high benefit of generalism $(\theta \gg 0)$, the sum of the resource carrying capacities is high enough to enable chaotic dynamics and even evolutionary suicide.

Figure 10 illustrates the case with $K_{1}>4$. This means that specialists with strategy sufficiently close to 1 will exhaust resource 1 . When resource 1 is exhausted, devoted specialists with strategy $s=1$ will vanish. If devoted specialists can invade the population and outcompete all the other strategies, evolutionary suicide may occur. However, there are several factors that may prevent evolutionary suicide in this setting. Most of all, when resource 1 is exhausted and devoted specialist wiped out, all the other strategies $(s<1)$ are, however, able to survive due to their ability to use resource 2 . The population sizes of almost devoted resource 1 specialists will, naturally, drop to very low values. However, since resource 1 recovers the next time unit after being exhausted, all the strategies that survived will start to increase in population size again. Furthermore, if $K_{1} \gg 4$, resource 1 is frequently exhausted already by specialists using strategy $s<1$. The more specialized an individual is (on resource 1) the more vulnerable the individual is to these occasions. This, in turn, may halt evolution such that the devoted specialism never enters the population. In other words, there exists an almost devoted specialist singular strategy in the regime of the chaotic population dynamics. (compare with Hoyle et al. (2011)). Even when devoted specialist are able to invade the population, it is not guaranteed that they can outcompete the other strategies $(s<1)$ before being wiped out by the next resource depletion. Even in the cases where evolutionary suicide might 
1 happen, it depends on the details of the simulation procedure whether evolutionary suicide is observed or not: In "the standard simulation procedure" used generally in the studies utilizing the adaptive dynamics approach (Kisdi, 1999; White and Bowers, 2005; White et al., 2006; Nurmi and Parvinen, 2008, 2011), the simulation step, where "extinct" strategies are removed from the population, is usually immediately followed by a step where a new mutant (resembling one of the extant strategies) is added to the population. This means that it is never possible for the devoted specialist strategy to be the only strategy present in the population when the population dynamics are iterated. If resource 1 is exhausted, the devoted specialists vanish and the population size of the newly added mutant becomes extremely small. However, this extremely rare mutant now constitutes the whole population and thus it won't be considered extinct in the "standart simulation procedure". In order to observe evolutionary suicide, it is necessary to add to the simulation procedure some additional iteration of the population dynamics (see the Appendix for details). Note that for evolutionary suicide to occur via generalist strategies, the situation is different (Figures 5B, 9B). There evolution directs towards generalism until both resources are exhausted simultaneously and all consumers are wiped out which happens for any reasonable simulation procedure.

When the evolution of a monomorphic population directs towards an evolutionary branching point, even cyclic evolution is possible. After branching, the two morphs evolve towards devoted specialism. When the branch specializing on resource 1 reaches the strategy $s=1$, it is wiped out due to the depletion of resource 1. The other branch remains intact, but since the population has become monomorphic, it starts to evolve towards generalism and a new branching results (compare with Kisdi et al. (2001); Dercole (2003)). This process is illustrated in Figure 10B. Note that cyclic evolution does not have an endpoint. Therefore in Figure 10A, we have chosen such a procedure for evolutionary simulations that evolutionary suicide is not possible. In Figure 10B, the simulation procedure is chosen such that evolutionary suicide is possible.

Note furthermore that, the mechanisms described above may, also in the evolutionary time-scale, enable the viability of populations that use two resources, even in environments where both devoted specialists strategies are unviable due to resource depletions caused by overly efficient resource usage. 


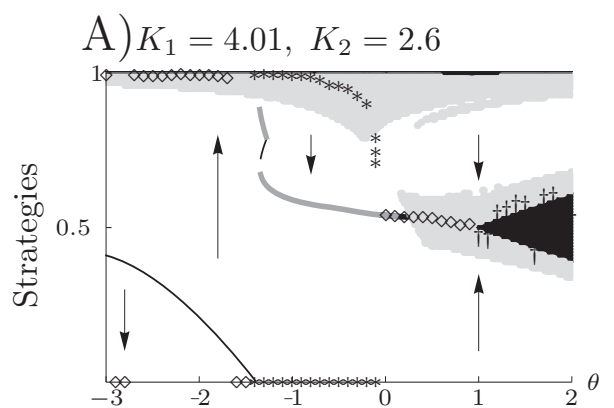

Trade-off parameter

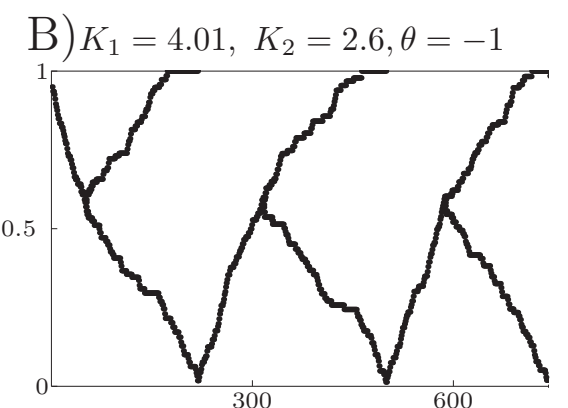

Evolutionary time

Figure 10: Evolutionary bifurcation diagram in the case when devoted resource 1 specialist is not viable due to resource depletion.

Panel A: Singular strategies and endpoints of evolutionary simulations as a function of the trade-off parameter $\theta$. The simulation procedure does not enable evolutionary suicide via specialism. Thin black curve indicates evolutionary repellors, thick grey curve branching points and thick black curve evolutionarily stable strategies. The arrows indicate the direction of evolution in a monomorphic population. In the black-colored parameter domain, the population is not viable. In the grey-colored parameter domain, the monomorphic population dynamics are (nearly) chaotic. If an evolutionary simulation ends in a monomorphic population, the end-strategy is denoted by $\diamond$. If it ends in a dimorphic or polymorphic population, the strategies comprising the endpoint are denoted by $*$-signs. If evolutionary suicide occurs, the last viable strategy is denoted by $\dagger$-sign.

Panel B: Strategies present in the population as a function of the evolutionary time when the simulation procedure enables evolutionary suicide via specialism. One unit of the evolutionary time corresponds to one loop of the simulation procedure depicted in the Appendix. Thus, it is only applicable for comparison between different simulations using the same procedure) Other parameter values: $\alpha_{1}=\alpha_{2}=1, \lambda_{1}=\lambda_{2}=1$. 


\section{Evolutionary dynamics in other mechanistically underpinned pop- ulation models}

Following Geritz and Kisdi (2004), also other resource dynamics than the logistic differential equation (1) can be used to obtain discrete-time population models for the consumer. If the resources, in the absence of consumers, grow according to the Gompertz equation

$$
\dot{R}_{i}=\alpha_{i}\left(\operatorname{Ln}\left(K_{i}\right)-\operatorname{Ln}\left(R_{i}\right)\right) R_{i},
$$

7 one obtains the famous Ricker (1954) model that, in the case of two resources

8 (with $\alpha_{1}=\alpha_{2}=1$ ) and $k$ consumer strategies, has the form

$$
\begin{aligned}
x_{n+1}^{(j)} & =\lambda_{1} K_{1} \beta\left(s^{(j)}\right) x_{n}^{(j)} \exp \left(-\sum_{i=1}^{k} \beta\left(s^{(i)}\right) x_{n}^{(i)}\right) \\
& +\lambda_{2} K_{2} \beta\left(1-s^{(j)}\right) x_{n}^{(j)} \exp \left(-\sum_{i=1}^{k} \beta\left(1-s^{(i)}\right) x_{n}^{(i)}\right),
\end{aligned}
$$

where the notations correspond to the ones in equation (3). Analogously, one can derive, for example, different variants of Hassell (1975) or Beverton and Holt (1957) models. Beverton-Holt model that shows only equilibrium dynamics has been extensively analyzed by Nurmi and Parvinen (2008). We analyzed the evolutionary dynamics also in Hassell model (not illustrated). According to our observations, they are qualitatively similar to those of the Ricker model (illustrated below). Note, that our parametrization of the Gompertz equation (7) is slightly different from the parametrization used by Geritz and Kisdi (2004). In the parametrization used by Geritz and Kisdi (2004), it is not straightforward to interpret the parameters $K_{i}$ as resource carrying capacities. This would unnecessarily complicate the evolutionary analysis and hence we have chosen to use parametrization (7) resulting in model (8).

Under equilibrium population dynamics, the evolutionary dynamics in the Ricker model are qualitatively similar to those of the logistic model (illustrated in Figure 2). Figure 11 illustrates the evolutionary dynamics in the Ricker model in the case of possibly periodic or chaotic population dynamics. The evolutionary dynamics are mainly qualitatively similar to those of the logistic model (illustrated in Figure 5). There are, however, some differences. The most obvious one is that the complete exhaustion of the resources is not possible in the Ricker model (compare equations (3) and (8)). Thus, deterministic evolutionary suicide is not possible. However, if demographic stochasticity was involved, evolutionary suicide would be possible. Another rather apparent difference between Figures $5 \mathrm{~B}$ and 11 is that the bifurcation structure in the parameter domain where the generalist strategy turns from a repellor into a branching point, is different, with Ricker model showing even three alternative branching points for the same trade-off parameter value (e.g. $\theta=-4.5)$. However, similar bifurcation structure can be found in the logistic model, as well, with slightly different parameter values (e.g. $\lambda=1.1$ and other parameters as in Figure 5B). 
Similarly to the logistic model, also in the Ricker model, there exists a pa2 rameter domain $(-2.7 \lesssim \theta \lesssim 0)$ where evolutionary branching first takes place and then the dimorphic population evolves neither to any singular strategy combination nor to devoted specialism. Instead, the strategies of the dimorphic population fluctuate in an intermediate strategy region, because chaotic population dynamics result in frequent population-dynamical attractor switchings that affect the evolutionary dynamics. When $-3.6 \lesssim \theta \lesssim-2.9$, another evolutionary branching takes place and evolution leads to the trimorphic coexistence of generalists and specialists. These phenomena are qualitatively similar to those observed in the logistic model even though they occur in different parameter domains. However, the transition between these two phenomena is different. In the logistic model, the transition occurs instantly without any intermediate phenomena, whereas in the Ricker model, there exists an intermediate parameter domain $(\theta \approx-2.8)$. In this intermediate parameter domain, second evolutionary branching takes place, but the appearance of the generalist strategy, however, affects the population dynamics such that the generalist dies out. When the 17 population becomes dimorphic again, it evolves back to the singular dimorphic 18 strategy pair and a new evolutionary branching takes place. This results in 19 cyclic evolution as illustrated in Figure 12. 


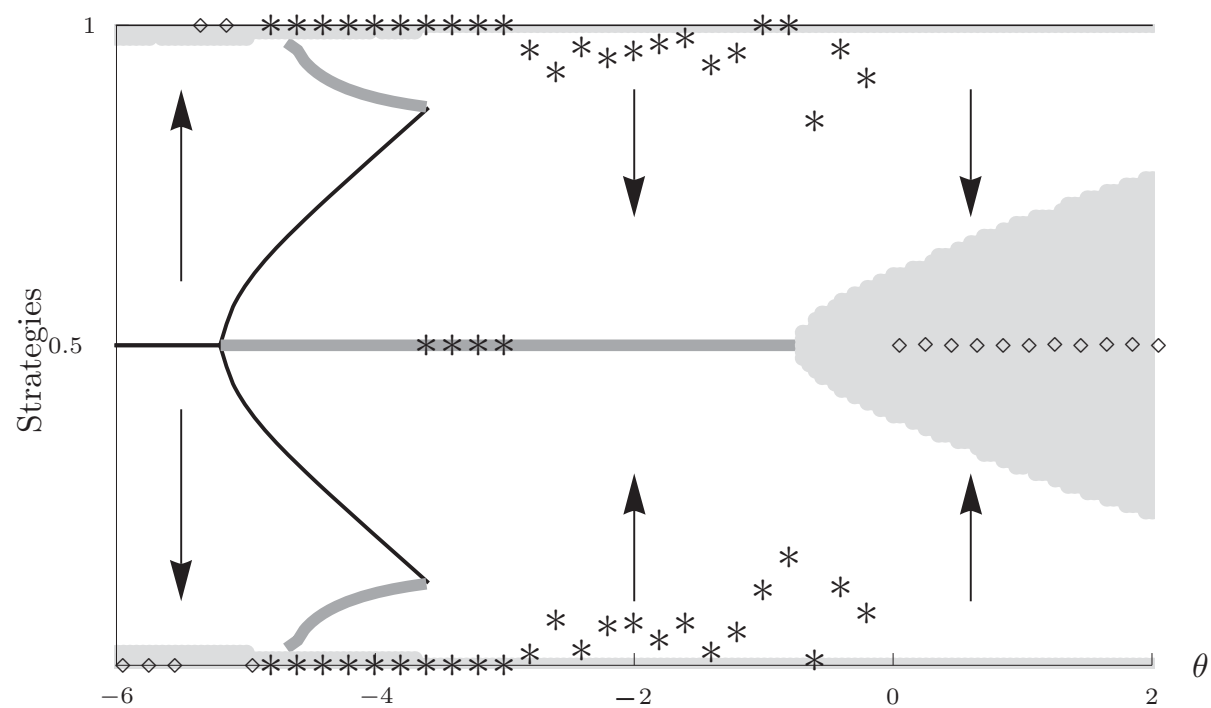

Figure 11: Evolutionary bifurcation diagram of the Ricker model with possibly chaotic population dynamics. Singular strategies and endpoints of evolutionary simulations as a function of the trade-off parameter $\theta$. Thin black curve indicates evolutionary repellors, thick grey curve branching points and thick black curve evolutionarily singular strategies. The arrows indicate the direction of evolution in a monomorphic population. In the grey-colored parameter domain, the monomorphic population dynamics are (nearly) chaotic. If an evolutionary simulation ends in a monomorphic population, the end-strategy is denoted by $\diamond$, else the strategies comprising the endpoint are denoted by $*$-signs.

Other parameter values: $K_{1}=18, K_{2}=18, \lambda_{1}=\lambda_{2}=1, \alpha_{1}=\alpha_{2}=1$. 
A) Strategies present during the evolutionary time

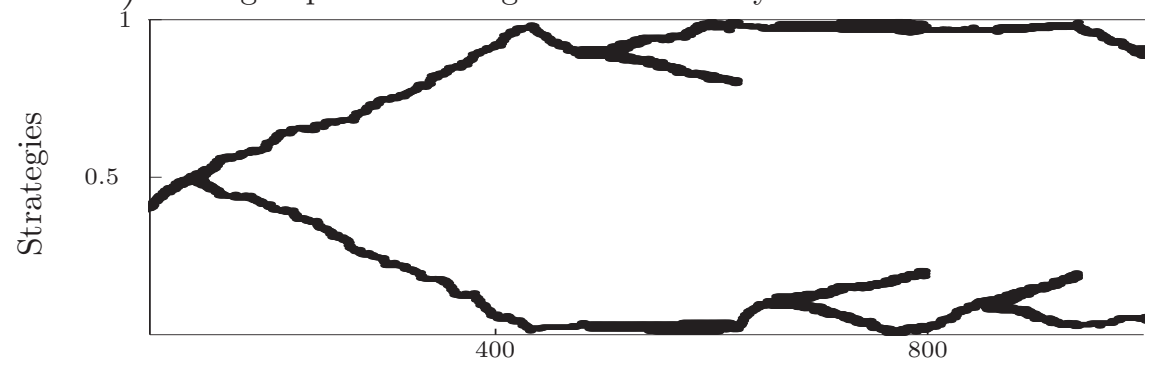

B) Sum of the resource availabilities

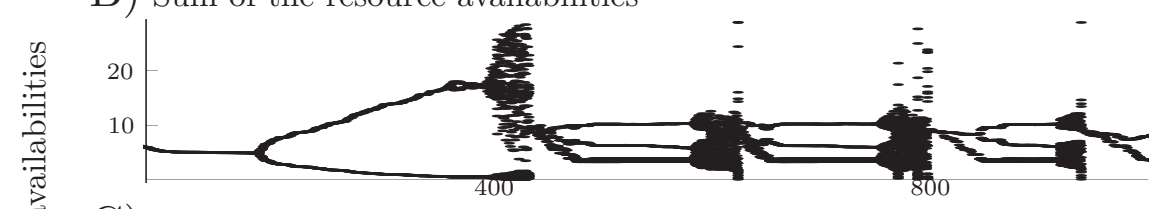

C) Difference between the resource availabilities

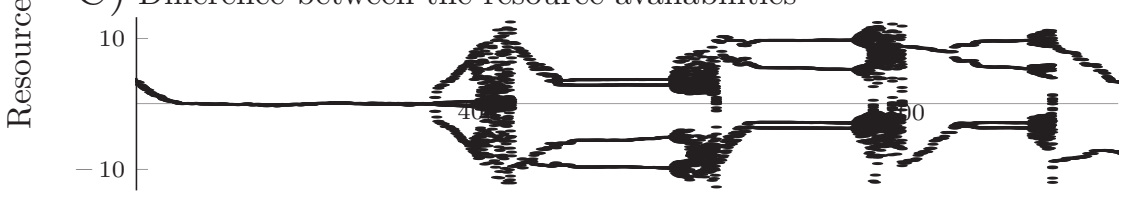

Evolutionary time

Figure 12: The result of a cyclic evolutionary simulation in Ricker model.

Panel A: Strategies present in the population as a function of the evolutionary time. One unit of the evolutionary time corresponds to one loop of the simulation procedure depicted in the Appendix. Thus, it is only applicable for comparison between different simulations using the same procedure. Initial population is monomorphic practicing strategy $s=0.4$.

Panels B and C: Resource availabilities $A_{1}$ and $A_{2}$ (for the logistic model corresponding availabilities are defined in equation (6)). For each evolutionary time unit, panel B illustrates the sum of the resources availabilities during each step on the population-dynamical attractor. Panel C illustrates the differences of the resource availabilities on the population-dynamical attractor for each evolutionary time unit.

Parameter values as in Figure 11 with $\theta=-2.8$. 


\section{Discussion}

In this paper, we have examined the evolution of resource specialization under non-equilibrium population dynamics. We use a model that is mechanistically underpinned on individual level ingredients, which makes it suitable for evolutionary analysis (Rueffler et al., 2006a). Furthermore, for a mechanistically underpinned model, it is easy to interpret parameters biologically, as well as consider, whether the model assumptions are satisfied in different biological scenarios. On the other hand, our model involves several simplifications that may affect model predictions:

- In our evolutionary analysis, we focus exclusively on the resource consumption rates $\beta$ and assume that consumers use resources according the law of mass-action with these rates. This allows us to focus on the evolutionary effects of non-equilibrium population dynamics. However, the model would be biologically more realistic if non-linear functional response was assumed, or if the behavioral elements affecting the evolution of specialization were taken into account (Rueffler et al., 2007; Abrams, 2010).

- Our previous results concerning the evolution of specialization under equilibrium metapopulation dynamics (Nurmi and Parvinen, 2008, 2011) suggest a strong interplay between dispersal and specialization. Thus, one should study also the consequences of non-equilibrium population dynamics for the joint evolution of dispersal and specialization in metapopulation models.

- We assume rather simple resource dynamics. However, the evolutionary changes in the consumer strategies are likely to cause evolutionary changes also in the resource populations resulting in co-evolution of resources and consumers (see e.g. Abrams (2000) and references therein).

- Our evolutionary analysis is based on clonal reproduction and the rather simple genetic architecture assumed by the adaptive dynamics approach. There are several studies indicating that the phenotypic models of evolution are capable to predict the course of evolution also in monomorphic sexually reproducing populations (Weissing, 1996; Kisdi and Geritz, 1999a,b; Geritz and Kisdi, 2000). However, when the monomorphic population encounters disruptive selection, sexual reproduction usually hinders branching in the absence of assortative mating or some other source of reproductive isolation (Dieckmann and Doebeli, 1999; Geritz and Kisdi, 2000; Doebeli et al., 2007).

- We have studied a large family of resource consumption functions with different curvature. However, the function is always either everywhere concave or everywhere convex. Trade-offs play an essential part in the evolution of specialization. Therefore, the usage of trade-off independent methods (de Mazancourt and Dieckmann, 2004; Bowers et al., 2005; Geritz 
et al., 2007) could reveal useful additional information concerning the evolutionary dynamics.

Under equilibrium population dynamics in a well-mixed population, the evolution of specialization, in the case of two alternative resources, always leads to a monomorphic generalist population when the resource consumption function is concave (trade-off weak, $\theta>0$ ) and to a population comprising one or two specialist strategies when the resource consumption function is convex (trade-off strong, $\theta<0$ ) (Levins, 1962, 1963; Schreiber and Tobiason, 2003; Ma and Levin, 2006; Nurmi and Parvinen, 2008; Zu et al., 2011a,b). Our results show that under non-equilibrium population dynamics this result no longer holds (even evolution to the trimorphic coexistence is possible). White et al. (2006) and Hoyle et al. (2011) have reached a similar conclusion when analyzing the case of trade-off between reproduction and survival. In fact, the results by Nurmi and Parvinen (2008) indicate, that also spatial (metapopulation) structure may break the relation between concavity of the resource consumption function and evolution to a monomorphic generalist population.

Furthermore, White et al. (2006) and Hoyle et al. (2011) showed that additional singular strategies may appear due to the non-equilibrium dynamics. Similar phenomena is present in our model: evolution to the dimorphic singular strategy pair (Figure 6), as well as evolution to the trimorphic coexistence (Figure 8) are evolutionary scenarios that are not possible under equilibrium population dynamics in a well-mixed population. Furthermore under nonequilibrium population dynamics, cyclic evolution is possible (Figures 10 and 12 ), and evolution may even lead to a dimorphic population with evolutionary fluctuations, where chaotic population dynamics enable population-dynamical attractor switchings that affect the evolutionary forces such that the dimorphic population evolves to another nearby intermediate strategy combination (Figure 7).

Hoyle et al. (2011) committed also thorough algebraic analysis of the transition from equilibrium to periodic population dynamics. Unfortunately, both of the phenomena we observe involve dimorphic populations and chaotic population dynamics. This prevents the in-depth analysis in the spirit of Hoyle et al. (2011). Especially, the interplay between evolutionary and ecological dynamics resulting in evolutionary fluctuations as depicted in Figure 7 would be interesting, since it seems that rare randomly occurring mutations may disturb the population dynamics and change the population-dynamical attractors such that also the evolutionary dynamics are affected, and the population evolves to another nearby strategy pair, but never to devoted specialism or to any singular strategy combination. Within the scope of this article, we can only present simulations indicating that such evolutionary scenarios exist; to fully understand them is an interesting task for further research.

Evolution starting from a monomorphic population may, under non-equilibrium population dynamics, also lead to the trimorphic coexistence of a generalist and two specialists. In such coexistence, each of the specialists uses the corresponding resource more efficiently than the competing strategies. The viability of 
the generalist strategy, on the other hand, is based on the asynchronous nonequilibrium population dynamics of the specialists. The population sizes of the specialist strategies fluctuate, and hence they are repeatedly rather low, which means that the corresponding resource is abundantly available allowing the generalist to increase in population size. This phenomenon was originally observed by Abrams (2006a) in a continuous-time model involving Holling type II functional response in the case where the dynamics of the two resources are different, which creates sufficient asynchrony to the resource dynamics. However, nonlinear functional response is known to have an essential part in allowing species coexistence, e.g., several species can coexist even on a single resource under non-equilibrium dynamics (Armstrong and McGehee, 1980; Kisdi and Liu, 2006; Geritz et al., 2007; Tachikawa, 2008). In our model, consumers use resources according to the law of mass-action with a linear functional response. Furthermore in our model, evolution to the trimorphic coexistence is possible also in the case of similar resources. Thus, our results indicate that non-equilibrium population dynamics really is the main factor enabling evolution to the trimorphic coexistence. As can be observed from Figure 5, evolution ends in the trimorphic coexistence in a rather small parameter domain. It is, however a robust phenomena that can be observed without fine-tuning of the parameters since it is a fundamental part of the evolutionary dynamics on chaotic population-dynamical attractors. It is always present in the parameter domain with transition from the evolution dynamics leading to generalism into evolution to the combination of the two devoted specialists.

It is also noteworthy that we observed evolution to the trimorphic coexistence only for such ecological scenarios where monomorphic devoted specialist populations have chaotic population dynamics. In these scenarios, the evolutionary path to the out-of-phase two-periodic population-dynamical orbit that allows the coexistence, always involves a series of period-doubling bifurcations and seemingly chaotic population dynamics. However, we are not aware of any theoretical reasons why chaotic population dynamics should necessarily be involved with the evolutionary path to the trimorphic coexistence, because symmetric attractors may become population-dynamically unstable even without chaos, and furthermore, the population-dynamical out-of-phase orbit is often stable in scenarios where the in-phase orbit is unstable.

Under equilibrium population dynamics, evolution leading to a singular dimorphic strategy pair comprising two partially specialized strategies is possible in well-mixed populations, but requires rather complicated forms of trade-off (Zu et al., 2011a,b). Under non-equilibrium dynamics, evolution to a singular dimorphic strategy pair is possible even when the resource consumption function is everywhere convex (see e.g. Figure 5A). In metapopulation models with equilibrium local dynamics, evolution to a singular strategy pair has been observed, but evolution to the trimorphic coexistence requires joint evolution with dispersal (Nurmi and Parvinen, 2008, 2011).

In our model with logistic resource dynamics, evolutionary suicide is possible when resources are abundantly available (high resource carrying capacities $K_{1}$ and/or $K_{2}$ ). Evolutionary suicide may take place via generalist strategies (e.g. 
1 Figure 5B) or via a specialist strategy (Figure 10). In the former case, both 2 resources are exhausted simultaneously, and all the strategies are wiped out. 3 This extinction occurs in all reasonable evolutionary simulation procedures. In 4 the latter case, only one resource is exhausted, and only the devoted specialists are wiped out whereas all the other strategies survive and start to grow in population size again, even though the population size may visit very low values. It depends on the details of the simulation procedure whether a devoted specialist is able to outcompete all the other strategies before being destroyed due to the depletion of the only resource it is able to utilize. Especially, evolutionary suicide is not possible in the "standard" simulation procedure used generally in adaptive dynamics studies (see the Appendix). When evolutionary suicide may take place via a specialist strategy, even evolutionary cycles of successive branching events and extinctions of one branch are possible (Figure 10). Note that the possibility of evolutionary suicide originates from the special characteristics of discrete logistic population dynamics. For example, the bifurcation diagram of the Ricker (as well as Hassell) equation (see equation (8)) is to large extent similar to the one of the logistic equation (see equation (2)), but there the resources are never completely exhausted, and evolutionary suicide is not possible in the absence of Allee-effects and demographic stochasticity (compare with Parvinen (2005)).

A large fraction of the studies combining evolutionary dynamics with fluctuating population dynamics have focused on co-evolution in predator-prey models (see e.g. a review by Abrams (2000)). Our model corresponds to these models in the case where only predator can evolve. According to Abrams (1992, 2000), the evolution of predator may sometimes cause predator-prey cycles, but usually it stabilizes the population dynamics. Our results contrast with this since the consumer evolution often destabilizes population dynamics. However, in line with our results, Abrams $(1992,2000)$ concluded that increasing prey/resource carrying capacity increased the probability for non-equilibrium population dynamics. In fact, our results relate to the paradox of enrichment (Rosenzweig, 1971), since increasing resource carrying capacity destabilizes population dynamics and may even cause extinction.

To sum up, we have systematically analyzed the evolution of specialization in resource utilization under non-equilibrium population dynamics. We have shown, that several evolutionary phenomena that are not possible under equilibrium population dynamics, may be possible under non-equilibrium population dynamics: additional singular dimorphic strategy pairs may appear, and furthermore, a secondary evolutionary branching may occur and evolution may lead to the trimorphic coexistence of two specialist strategies and a generalist strategy.

\section{A. Simulation procedure}

The simulations we commit are not individual based simulations. Instead, 3 we iterate the population dynamics (3) with infrequent insertions of new mutant 4 strategies and removals of strategies that have diminished sufficiently in order 
to be considered extinct. The simulation procedure differs slightly from the assumptions used by the adaptive dynamics machinery since simulations are not completely mutation limited as new mutants may appear before the population has reached an attractor. The simulation procedure is:

1. Iterate the population dynamics according to the equation (3). Here this is typically done 2000 times.

2. Remove all the strategies that can be considered extinct. Note, that under chaotic population dynamics, it is difficult to determine which strategies to remove since the whole population may occasionally be very small. We have chosen to base the removal of a strategy on its relative proportion of the entire population. When strategies go extinct due to resource depletion this is not a problem because in this case the population size is exactly zero. Here we typically consider a strategy extinct if its population size is less than 0.0001 times the total population size.

3. In order to speed up calculations, we set a maximum number of strategies present in the simulation. If the number of strategies is not below this limit, go back to step 1. Here the maximum number of strategies was typically 20. However, this almost never affects results.

4. Pick one strategy that will mutate. The probability to pick a certain strategy is determined by the strategy's population size divided by the total population size.

5. Pick the size of the mutation using uniform distribution $\mathrm{U}[-m, m]$, where $m$ is the largest possible mutation. Here typically $m=0.01$.

6. Check that the new strategy belongs to the strategy space and differs sufficiently from the extant strategies. If not, go back to step 4. Several occurrences of almost similar strategies in a simulation would unnecessarily slow down the simulation. We usually require minimum difference 0.001 .

(7.) Iterate the population dynamics.

(8.) Remove the extinct strategies. Now it is possible that the population becomes monomorphic comprising devoted specialists only.

(9.) Iterate the population dynamics. Now it is possible that a resource depletion wipes out devoted specialists and the population goes extinct.

10. Continue from step 1 if the population persists.

One loop of this procedure corresponds to one unit of the evolutionary time in the figures that illustrate results of evolutionary simulations (Figures 1, 8, 10 and 12). In these figures, the illustrated length of the evolutionary time has been chosen such that the figures illuminate the question in focus in the optimal way. The actual simulations did run much longer than the illustrated 
evolutionary time interval in order ensure that the system has really reached its evolutionary endpoint (which can never be guaranteed, however).

When steps 7-9 are not included in the simulation procedure, it corresponds to the "standard adaptive dynamics simulation" (Kisdi, 1999; White and Bowers, 2005; White et al., 2006; Nurmi and Parvinen, 2008, 2011) and evolutionary suicide via specialism is not possible since the population never comprises solely devoted specialists. Assume that the devoted specialists can outcompete the other strategies in step 1. The other strategies are removed in step 2. However, immediately in steps 3-6 a new mutant enters the population. Thus, when the population dynamics are iterated again in step 1, the population comprises devoted specialists and a rare mutant that is also a specialist, but not a devoted one. If one resource is exhausted, the devoted specialists vanish. The mutant population becomes extremely small, but it survives because of its ability to use the other resource. However, this extremely rare mutant now constitutes the entire population and it will not be removed in step 2. Devoted specialists may later again outcompete this strategy, but devoted specialists can never constitute the whole population. Thus, evolutionary suicide via specialism is possible only when steps 7-9 are included to the simulation procedure. In step 7 devoted specialists may outcompete other strategies that are then removed in step 8, leaving only devoted specialists alive. If resource depletion is encountered during the iteration of the population dynamics, devoted specialists are wiped out and thus the whole population goes extinct.

Note that in step 2, we use a relative extinction threshold, i.e., we declare a strategy extinct if its population size is small compared to the size of the entire population. Alternatively, we could have used an absolute extinction threshold, i.e., declare a strategy extinct when its population size is small (independent of the size of the entire population). The type of the extinction threshold affects essentially the possibility of evolutionary suicide as well as the evolutionary dynamics under chaotic population dynamics. Using an absolute extinction threshold involves the implicit assumption of demographic stochasticity: once a population becomes extremely small, it will be wiped out by demographic stochasticity. An absolute extinction threshold, although it might be biologically more realistic, is problematic when combined with chaotic population dynamics, since the chaotic population-dynamical fluctuations may then drive otherwise viable strains or the entire population to extinction, and thus the size of the extinction threshold might affect the evolutionary dynamics. Moreover, in this paper, we considered the possibility for deterministic evolutionary suicide, and hence we need to exclude stochasticity. Thus, we have chosen to use a relative extinction threshold. It is noteworthy that this may slightly favor increased efficiency in average resource usage (generalists in Figures 5, 9B, and 11, resource 1 specialists in Figure 10). 


\section{B. Algebraic proof: Dimorphic evolution on symmetric population- dynamical attractors directs towards devoted specialism.}

Assume that the environment is symmetric with $K_{1}=K_{2}=K, \lambda_{1}=\lambda_{2}=$ $\lambda, \alpha_{1}=\alpha_{2}=\alpha$ and the population is dimorphic with strategies $s^{(1)}=s^{\text {res }}$ and $s^{(2)}=1-s^{\text {res }}$, where $s^{\text {res }}<0.5$. Assume further that the dimorphic population is on a symmetric attractor such that $x_{n}^{(1)}=x_{n}^{(2)}=x_{n}^{\text {res }}$ for each time unit $n$. Then, for a mutant with strategy $s^{\text {mut }}$ and population size $x_{n}^{\text {mut }}$, the equation s (3) determining fecundity has the form $x_{n+1}^{\text {mut }}=f\left(s^{\text {mut }}, S^{\text {res }}, X_{n}^{\text {res }}\right) x_{n}^{\text {mut }}$, where

$$
\begin{aligned}
f\left(s^{\mathrm{mut}}, S^{\mathrm{res}}, X_{n}^{\mathrm{res}}\right)= & K \max \left\{0,1-x_{n}^{\mathrm{res}}\left(\beta\left(s^{\mathrm{res}}\right)+\beta\left(1-s^{\mathrm{res}}\right)\right)\right\} \\
& \cdot\left(\beta\left(s^{\mathrm{mut}}\right)+\beta\left(1-s^{\mathrm{mut}}\right)\right) \\
= & H\left(S^{\mathrm{res}}, X_{n}^{\mathrm{res}}\right)\left(\beta\left(s^{\mathrm{mut}}\right)+\beta\left(1-s^{\mathrm{mut}}\right)\right),
\end{aligned}
$$

9 where $S^{\text {res }}=\left(\begin{array}{c}s^{\text {res }} \\ 1-s^{\text {res }}\end{array}\right), X_{n}^{\text {res }}=\left(\begin{array}{c}x_{n}^{\text {res }} \\ x_{n}^{\text {res }}\end{array}\right)$, and $H\left(S^{\text {res }}, X_{n}^{\text {res }}\right)$ stands for a shorthand notation for all the terms that do not involve the mutant strategy $s^{\text {mut }}$. Other mechanistically underpinned models, such as (8), result in analogous expressions with a different function $H$. Therefore, the following applies to other models as well. The fitness of a mutant in the environment determined by this dimorphic symmetric resident population is, according to the equation (4),

$$
\begin{aligned}
r\left(s^{\mathrm{mut}}, S^{\mathrm{res}}, X^{\mathrm{res}}\right) & =\lim _{t \rightarrow \infty} \frac{1}{t} \sum_{i=1}^{t} \ln \left(f\left(s^{\mathrm{mut}}, S^{\mathrm{res}}, X_{i}^{\mathrm{res}}\right)\right) \\
& =\ln \left(\beta\left(s^{\mathrm{mut}}\right)+\beta\left(1-s^{\mathrm{mut}}\right)\right) \\
& +\lim _{t \rightarrow \infty} \frac{1}{t} \sum_{i=1}^{t} \ln \left(H\left(S^{\mathrm{res}}, X_{i}^{\mathrm{res}}\right)\right) .
\end{aligned}
$$

Now it is already obvious that the fitness function is convex whenever the resource consumption function $\beta$ is convex $(\theta<0)$. Furthermore, the fitness 8 gradient at the strategy $s^{\text {res }}$ is

$$
\left.\frac{\partial r}{\partial s^{\mathrm{mut}}}\right|_{s^{\mathrm{mut}}=s^{\mathrm{res}}}=\frac{\beta^{\prime}\left(s^{\mathrm{res}}\right)-\beta^{\prime}\left(1-s^{\mathrm{res}}\right)}{\left(\beta\left(s^{\mathrm{res}}\right)+\beta\left(1-s^{\mathrm{res}}\right)\right)},
$$

which is negative for a convex resource consumption function since we assumed that $s^{\text {res }}<0.5$. Thus the strategy $s^{\text {mut }}$ can invade and outcompete strategy $s^{\text {res }}$ if and only if $s^{\text {mut }}<s^{\text {res }}$. Analogously, the fitness gradient at the strategy $1-s^{\text {res }}$ is

$$
\left.\frac{\partial r}{\partial s^{\mathrm{mut}}}\right|_{s^{\mathrm{mut}}=\left(1-s^{\mathrm{res}}\right)}=\frac{\beta^{\prime}\left(1-s^{\mathrm{res}}\right)-\beta^{\prime}\left(s^{\mathrm{res}}\right)}{\left(\beta\left(s^{\mathrm{res}}\right)+\beta\left(1-s^{\mathrm{res}}\right)\right)}
$$


which is positive for a convex resource consumption function since $1-s^{\mathrm{res}}>0.5$. Thus the strategy $s^{\text {mut }}$ can invade and outcompete strategy $1-s^{\text {res }}$ if and only if $s^{\text {mut }}>1-s^{\text {res }}$.

Altogether, the two branches will evolve further away from the unbiased generalist strategy until devoted specialism is reached, or the symmetry of strategies is lost, or the symmetry of the population-dynamical attractors is lost.

\section{References}

Abrams, P., 2006a. Adaptive Change in the Resource-Exploitation Traits of a Generalist Consumer: The Evolution and Coexistence of Generalist and Specialists. Evolution 60, (427-439).

Abrams, P., 2006b. The Prerequisities for and Likelihood of Generalist-Specialist Coexistence. Am. Nat. 167, (329-342).

Abrams, P., 2010. Quantitative descriptions of resource choice in ecological models. Popul. Ecol. 52, (47-58).

Abrams, P. A., 1992. Adaptive foraging by predators as a cause of predator-prey cycles. Evol. Ecol. 6, (56-72).

Abrams, P. A., 2000. The evolution of predator-prey interactions: Theory and evidence. Annu. Rev. Ecol. Syst. 31, (79-105).

Armstrong, R., McGehee, R., 1980. Competitive Exclusion. Am. Nat. 115, (151170).

Beverton, R., Holt, S., 1957. On the Dynamics of Exploited Fish Populations. Vol. 19 of Fisheries Investigations, Series 2. H.M. Stationery Office, London.

Bowers, R. G., Hoyle, A., White, A., Boots, M., 2005. The geometric theory of adaptive evolution: trade-off and invasion plots. J. Theor. Biol. 233, (363$377)$.

de Mazancourt, C., Dieckmann, U., 2004. Trade-off geometries and frequencydependent selection. Am. Nat. 164, (765-778).

Debarre, F., Lenormand, T., 2011. Distance-limited dispersal promotes coexistence at habitat boundaries: reconsidering the competitive exclusion principle. Ecol. Lett. 14, (260-266).

Dercole, F., 2003. Remarks on branching-extinction evolutionary cycles. J. Math. Biol. 47, (569-580).

Dercole, F., Ferriere, R., Rinaldi, S., 2002. Ecological Bistability and Evolutionary Reversals Under Asymmetrical Competition. Evolution 56, (1081-1090).

5 Dieckmann, U., Doebeli, M., 1999. On the origin of species by sympatric speciation. Nature 400, 354-357. 
Doebeli, M., Blok, H. J., , Leimar, O., Ulf, D., 2007. Multimodal pattern formation in phenotype distributions of sexual populations. Proc. R. Soc. Lond. B 274, (347-357).

Egas, M., Dieckmann, U., Sabelis, M., 2004. Evolution restricts the Coexistence of Specialists and Generalists: The Role of the Trade-off Structure. Am. Nat. 163, (518-531).

Ferrière, R., 2000. Adaptive responses to environmental threats: evolutionary suicide, insurance, and rescue. Options Spring 2000, IIASA, Laxenburg, Austria, $12-16$.

Geritz, S. A. H., Gyllenberg, M., Jacobs, F. J. A., Parvinen, K., 2002. Invasion Dynamics and Attractor Inheritance. J. Math. Biol. 44, (548-560).

2 Geritz, S. A. H., Kisdi, É., 2000. Adaptive Dynamics in Diploid, Sexual Populations and the Evolution of Reproductive Isolation. Proc. R. Soc. Lond. B 267, (1671-1678).

Geritz, S. A. H., Kisdi, É., 2004. On the mechanistic underpinning of discretetime population models with complex dynamics. J. Theor. Biol. 228, (261269).

Geritz, S. A. H., Kisdi, É., Meszéna, G., Metz, J. A. J., 1998. Evolutionary Singular Strategies and the Adaptive Growth and Branching of the Evolutionary Tree. Evol. Ecol. 12, (35-57).

Geritz, S. A. H., Kisdi, É., Yan, P., 2007. Evolutionary branching and long-term coexistence of cycling predators: Critical function analysis. Theor. Popul. Biol. 71, (424-435).

Gyllenberg, M., Parvinen, K., Dieckmann, U., 2002. Evolutionary Suicide and Evolution of Dispersal in Structured Metapopulations. J. Math. Biol. 45, (79$105)$.

Gyllenberg, M., Söderbacka, G., Ericsson, S., 1993. Does Migration Stabilize Local Population Dynamics? Analysis of a Discrete Metapopulation Model. Math. Biosci. 118, (25-49).

Hardin, G., 1968. The Tragedy of Commons. Science 162, (1243-1224).

Hassell, M., 1975. Density-dependence in single-species populations. J. Anim. Ecol. 44, (283-295).

Holmgren, R. A., 1994. A First Course in Discrete Dynamical Systems. SpringerVerlag.

Holt, R. D., McPeek, M., 1996. Chaotic population dynamics favors the evolution of dispersal. Am. Nat. 148, (709-718). 
1 Hoyle, A., Bowers, R. G., White, A., 2011. Evolutionary Behaviour, Trade-Offs and Cyclic and Chaotic Population Dynamics. Bull. Math. Biol. 73, (11541169).

4 Karonen, I., 2011. Stable coexistence in a lattice model of spatial competition with two site types. J. Theor. Biol. 295, (77-85).

6 Kisdi, É., 1999. Evolutionary branching under asymmetric competition. J. Theor. Biol. 197, (149-162).

8 Kisdi, É., 2002. Dispersal: Risk Spreading versus Local Adaptation. Am. Nat. $159,(579-596)$.

Kisdi, É., Geritz, S. A. H., 1999a. Adaptive Dynamics in Allele Space: Evolution of Genetic Polymorphism by Small Mutations in a Heterogeneous Environment. Evolution 53, (993-1008).

Kisdi, É., Geritz, S. A. H., 1999b. Evolutionary Branching and Sympatric Speciation in Diploid Populations. IIASA Studies in Adaptive Dynamics 39, (1-22).

Kisdi, É., Jacobs, F., Geritz, S. A. H., 2001. Red Queen evolution by cycles of evolutionary branching and extinction. Selection 2, (161-176).

Kisdi, É., Liu, S., 2006. Evolution of handling time can destroy the coexistence of cycling predators. J. Evol. Biol. 19, (49-58).

Levins, R., 1962. Theory of Fitness in a Heterogeneous Environment. I. The Fitness Set and Adaptive Function. Am. Nat. 96, (361-373).

Levins, R., 1963. Theory of Fitness in a Heterogeneous Environment. II. Developmental Flexibility and Niche Selection. Am. Nat. 97, (75-90).

Ma, J., Levin, S., 2006. The Evolution of Resource Adaption: How Generalist and Specialist Consumers Evolve. Bull. Math. Biol. 68, (1111-1123).

Maynard Smith, J., Price, G. R., 1973. The Logic of Animal Conflict. Nature $246,(15-18)$.

Meszéna, G., Czibula, J., Geritz, S. A. H., 1997. Adaptive Dynamics in a 2patch environment: a toy model for allopatric and parapatric speciation. J. Biol. Syst. 5, (265-284).

Metz, J. A. J., Nisbet, R., Geritz, S. A. H., 1992. How Should We Define Fitness for General Ecological Scenarios? Trends Ecol. Evol. 7, (198-202).

Nurmi, T., Geritz, S. A. H., Parvinen, K., Gyllenberg, M., 2008. Evolution of Specialization on Resource Utilization in Structured Metapopulations. J. Biol. Dyn. 2, (297-322).

Nurmi, T., Parvinen, K., 2008. On the evolution of specialization with a mechanistic underpinning in metapopulations. Theor. Pop. Biol. 73, (222-243). 
1 Nurmi, T., Parvinen, K., 2011. Joint evolution of specialization and dispersal in structured metapopulations. J. Theor. Biol. 275, (78-92).

Parvinen, K., 1999. Evolution of migration in a metapopulation. Bull. Math. Biol. 61, (531-550).

5 Parvinen, K., 2005. Evolutionary suicide. Acta Biotheor. 53, (241-264).

- Ravigné, V., Dieckmann, U., Olivieri, I., 2009. Live Where You Thrive: Joint Evolution of Habitat Choice and Local Adaptation Facilitates Specialization and Promotes Diversity. Am. Nat. 174, (E141-E169).

Ricker, W., 1954. Stock and recruitment. J. Fish. Res. Bd. Canada 11, (559$623)$.

Ronce, O., 2007. How Does It Feel to Be Like a Rolling Stone? Ten Questions About Dispersal Evolution. Annu. Rev. Ecol. Syst. 38, (231-253).

Rosenzweig, M., 1971. Paradox of enrichment: destabilization of exploitation ecosystems in ecological time. Science 171, (385-387).

Rueffler, C., Egas, M., Metz, J., 2006a. Evolutionary Predictions Should Be Based on Individual-Level Traits. Am. Nat. 168, (E148-E162).

Rueffler, C., Van Dooren, T. J., Metz, J., 2006b. The evolution of Resource Specialization through Frequency-Dependent and Frequency-Independent Mechanisms. Am. Nat. 167, (81-93).

Rueffler, C., Van Dooren, T. J., Metz, J., 2007. The Interplay between Behavior and Morphology in the Evolutionary Dynamics of Resource Specialization. Am. Nat. 169, (E34-E52).

Schreiber, S., Tobiason, G., 2003. The Evolution of Resource Use. J. Math. Biol. $47,(56-78)$.

Tachikawa, M., 2008. Fluctuation induces evolutionary branching in a mathematical model of ecosystems. Plos ONE 3, e3925.

Weissing, F., 1996. Genetic versus phenotypic models of selection: can genetics be neglected in a long-term perspective. J. Math. Biol. 34, (533-555).

White, A., Bowers, R. G., 2005. Adaptive dynamics of Lotka-Volterra systems with trade-offs: the role of interspecific parameter dependence in branching. Math. Biosci. 193, (101-117).

White, A., Greenman, J., Benton, T., Boots, M., 2006. Evolutionary behaviours in ecological systems with trade-offs and non-equilibrium population dynamics. Evol. Ecol. Res. 8, (387-398).

Wilson, D. S., Yoshimura, J., 1994. On the Coexistence of Specialists and Generalists. Am. Nat. 144, (692-707). 
1 Zu, J., Mimura, M., Takeuchi, Y., 2011a. Adaptive evolution of foraging-related 2 traits in a predatorprey community. J. Theor. Biol. 268, (14-29).

3 Zu, J., Wang, K., Mimura, M., 2011b. Evolutionary branching and evolutionarily stable coexistence of predator species: Critical function analysis . Math. Biosci. 231, (210-224). 